\title{
Impacts of a power-law non-thermal electron tail on the ionization and recombination rates
}

\author{
D. Porquet, M. Arnaud, and A. Decourchelle
}

\author{
CEA/DSM/DAPNIA, Service d'Astrophysique, C.E. Saclay, 91191 Gif-sur-Yvette Cedex, France
}

Received 6 March 2001 / Accepted 27 April 2001

\begin{abstract}
We have investigated the effects of a non-thermal electron population on the ionization and recombination rates. The considered electron distribution is defined as a Maxwellian function below a break energy $E_{\mathrm{b}}$ and as a power-law function of index $\alpha$ above this energy. We have calculated the collisional (direct and excitation autoionization) ionization coefficient rates as well as the (radiative and dielectronic) recombination rates. Practical methods are given to calculate these rates in order to be easily included in a computer code. The ionization rates are very sensitive to the non-thermal electron population and can be increased by several orders of magnitude depending on the temperature and parameters of the power-law function $\left(E_{\mathrm{b}}\right.$ and $\left.\alpha\right)$. The non-thermal electrons have a much weaker effect on the (radiative and dielectronic) recombination rates. We have determined the mean electric charge of elements C, N, O, Ne, Mg, Si, S, Ar, Ca, Fe and Ni for different values of the break energy and power-law index. The ionization balance is affected significantly, whereas the effect is smaller in ionizing plasmas.
\end{abstract}

Key words. acceleration of particles - atomic data - atomic processes - radiation mechanisms: non-thermal shock waves - ISM: supernova remnants

\section{Introduction}

The ionization and recombination rates for astrophysical plasmas have usually been calculated for a Maxwellian electron distribution (e.g., Arnaud \& Rothenflug 1985; Arnaud \& Raymond 1992; Mazzotta et al. 1998). However, in many low-density astrophysical plasmas, electron distributions may differ from the Maxwellian distribution. The degree of ionization of a plasma depends on the shape of the electron distribution, as well as on the electronic temperature. This has been studied for the solar corona (e.g. Roussel-Dupré 1980; Owocki \& Scudder 1983; Dzifcáková 1992; Dzifcáková 1998) and for evaporating interstellar clouds (Ballet et al. 1989), where a non-thermal electron distribution occurs in places where there are high gradients of density or temperature.

A non-thermal electron population is expected in various astrophysical plasmas. Strong shocks can convert a large fraction of their energy into the acceleration of relativistic particles by the diffusive shock acceleration process (e.g., Drury 1983; Blandford \& Eichler 1987; Jones \& Ellison 1991; Kang \& Jones 1991). Direct evidence for the presence of accelerated electrons up to relativistic energies $(\simeq 1 \mathrm{GeV})$ comes from the observations of radio synchrotron emission in supernova remnants and in clusters of galaxies. More recently, non-thermal X-ray emission has been reported in several shell-like supernova remnants and

Send offprint requests to: D. Porquet, e-mail: dporquet@cea.fr interpreted as synchrotron radiation from cosmic-ray electrons up to $\simeq 100 \mathrm{TeV}$ (Koyama et al. 1995; Allen et al. 1997; Koyama et al. 1997; Slane et al. 1999; Slane et al. 2001).

A number of recent works have focused on the nonthermal emission from supernova remnants (e.g., Laming 2001; Ellison et al. 2000; Berezhko \& Völk 2000; Bykov et al. 2000b; Baring et al. 1999; Gaisser et al. 1998; Reynolds 1996, 1998; Sturner et al. 1997) and clusters of galaxies (e.g., Sarazin 1999; Bykov et al. 2000a; Sarazin \& Kempner 1999). The impact of efficient acceleration on the hydrodynamics and thermal X-ray emission has been investigated (Decourchelle et al. 2000; Hughes et al. 2000).

When the acceleration is efficient, the non-thermal population is expected to modify directly the ionization rates in the plasma as well as the line excitation (e.g. Dzifcáková 2000; Seely et al. 1987). A hybrid electron distribution (Maxwellian plus power-law tail) is expected from diffusive shock acceleration (e.g., Berezhko \& Ellison 1999; Bykov \& Uvarov 1999). The low energy end of the power-law electron distribution (which connects to the Maxwellian thermal population) is likely to enhance the ionization rates and to significantly modify the degree of ionization of the plasma, which is used as a diagnostic of the plasma electron temperature.

In this paper, we shall examine the influence of a power-law non-thermal electron distribution (connecting to the falling Maxwellian thermal population) on the ionization and recombination rates for $\mathrm{C}, \mathrm{N}, \mathrm{O}, \mathrm{Ne}, \mathrm{Mg}, \mathrm{Si}$, 
$\mathrm{S}, \mathrm{Ar}, \mathrm{Ca}, \mathrm{Fe}$ and Ni. For different characteristic values of the power-law electron distribution, the mean electric charge of these elements has been determined as a function of the temperature at ionization equilibrium and for different values of the ionization timescale. We intend, in this paper, to give a comprehensive study of the dependence of these quantities on the parameters of the nonthermal population, illustrated by simple examples. We do not provide tables, which would be too numerous as the ionization equilibrium depends in our model on four parameters (element, temperature of the thermal component, index and low energy break of the non-thermal population). In the appendix or directly in the text, we give the formula needed for the calculations of the rates which could be easily inserted in computer codes.

In Sect. 2, we define the Hybrid electron distribution used in this work. The calculation of the new ionization collisional rates and (radiative and dielectronic) recombination rates is discussed in Sect. 3. In Sect. 4, we present the derived mean electric charge of the elements in ionization equilibrium as well as in ionizing plasmas.

\section{The electron distribution shapes}

The Maxwellian distribution, generally considered for the electron distribution in astrophysical plasmas, $N_{\mathrm{e}}(E)$, is defined as:

$$
\begin{aligned}
\mathrm{d} N_{\mathrm{e}}(E) & =n_{\mathrm{e}} f_{\mathrm{E}}^{\mathrm{M}}(E) \mathrm{d} E \\
f_{\mathrm{E}}^{\mathrm{M}}(E) & =\frac{2}{\sqrt{\pi}}(k T)^{-3 / 2} E^{1 / 2} \mathrm{e}^{-\frac{E}{k T}}
\end{aligned}
$$

where $E$ is the energy of the electron, $T$ is the electronic temperature and $n_{\mathrm{e}}$ the total electronic density. In this expression the Maxwellian function $f_{\mathrm{E}}^{\mathrm{M}}(E)$ is normalised so that $\int_{0}^{\infty} f_{\mathrm{E}}^{\mathrm{M}}(E) \mathrm{d} E=1$.

It is convenient to express this distribution in term of the reduced energy $x=E / k T$ :

$$
\begin{aligned}
\mathrm{d} N_{\mathrm{e}}(x) & =n_{\mathrm{e}} f^{\mathrm{M}}(x) \mathrm{d} x \\
f^{\mathrm{M}}(x) & =\frac{2}{\sqrt{\pi}} x^{\frac{1}{2}} \mathrm{e}^{-x} .
\end{aligned}
$$

The corresponding scaled (non-dimensional) distribution $f^{\mathrm{M}}(x)$ is an universal function, of fixed shape.

Non-Maxwellian electron distributions expected in the vicinity of shock waves, as in young supernova remnants, seem to be reasonably described by a Maxwellian distribution at low energy up to a break energy $E_{\mathrm{b}}$, and by a power-law distribution at higher energy (e.g., Berezhko \& Ellison 1999; Bykov \& Uvarov 1999). We call hereafter this "Maxwellian/Power-law" type of electron distribution the Hybrid electron distribution $\left(f^{\mathrm{H}}\right)$. It is defined, in reduced energy coordinates, as:

$\mathrm{d} N_{\mathrm{e}}(x)=n_{\mathrm{e}} f^{\mathrm{H}}(x) \mathrm{d} x$

$$
\begin{array}{ll}
f^{\mathrm{H}}(x)=C\left(x_{\mathrm{b}}, \alpha\right) \frac{2}{\sqrt{\pi}} x^{1 / 2} \mathrm{e}^{-x} & x \leq x_{\mathrm{b}} \\
f^{\mathrm{H}}(x)=C\left(x_{\mathrm{b}}, \alpha\right) \frac{2}{\sqrt{\pi}} x_{\mathrm{b}}^{1 / 2} \mathrm{e}^{-x_{\mathrm{b}}}\left(\frac{x}{x_{\mathrm{b}}}\right)^{-\alpha} \quad x \geq x_{\mathrm{b}},
\end{array}
$$

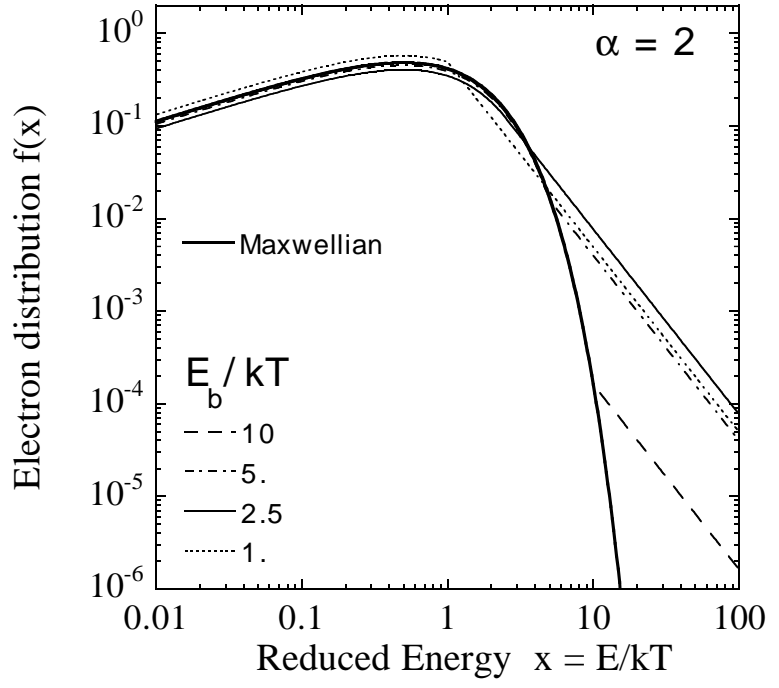

Fig. 1. The Hybrid (Maxwell/Power-law) electron distribution for different values of the break parameter $x_{\mathrm{b}}$, compared to a Maxwellian distribution $\left(x_{\mathrm{b}}=\infty\right)$. The slope of the powerlaw has been fixed to $\alpha=2$. Reduced energy coordinates, $x=E / k T$, are used.

where $x_{\mathrm{b}}=E_{\mathrm{b}} / k T$ is the reduced break energy, and $\alpha$ is the energy index of the power-law $(\alpha>1)$. Note that for $\alpha \leq 2$ the energy diverges (in practice a cutoff at very high energy occurs). Since for the calculations of the ionization and recombination rates the very high energies $(\geq 20 k T)$ have negligible effect, for simplicity, we use here a power-law defined from $x_{\mathrm{b}}$ to infinity.

The normalisation factor of the power-law distribution is defined so that the electron distribution is continous at $x_{\mathrm{b}}$. The factor $C\left(x_{\mathrm{b}}, \alpha\right)$ is a normalisation constant, so that $\int_{0}^{\infty} f^{\mathrm{H}}(x) \mathrm{d} x=1$ :

$C\left(x_{\mathrm{b}}, \alpha\right)=\frac{\sqrt{\pi}}{2} \frac{1}{\gamma\left(\frac{3}{2}, x_{\mathrm{b}}\right)+(\alpha-1)^{-1} x_{\mathrm{b}}^{3 / 2} \mathrm{e}^{-x_{\mathrm{b}}}}$

where $\gamma(a, x)$ is the gamma function defined as $\gamma(a, x)=$ $\int_{0}^{x} t^{a-1} \mathrm{e}^{-t} \mathrm{~d} t$. For $x \leq x_{\mathrm{b}}$, the Hybrid distribution only differs from a Maxwellian distribution by this multiplicative factor. The scaled distribution $f^{\mathrm{H}}(x)$ only depends on the two non-dimensional parameters, $x_{\mathrm{b}}$ and $\alpha$. The dependence on $k T$ of the corresponding physical electron distribution is $f_{\mathrm{E}}^{\mathrm{H}}(E)=(k T)^{-1} f^{\mathrm{H}}(E / k T)$.

The Hybrid distributions $f^{\mathrm{H}}(x)$, obtained for several values of the energy break $x_{\mathrm{b}}$, are compared to the Maxwellian distribution in Fig. 1. The slope has been fixed to $\alpha=2$, a typical value found in the models referenced above. The variation of the reduced median energy of the distribution with $x_{\mathrm{b}}$, for $\alpha=1.5,2 ., 3$., is plotted in Fig. 2, as well as the variation of the normalisation factor $C\left(x_{\mathrm{b}}, \alpha\right)$.

As apparent in the figures, there is a critical value of $x_{\mathrm{b}}$, for each $\alpha$ value, corresponding to a qualitative change in the behavior of the Hybrid distribution. This can be understood by looking at the distribution at the break energy $x_{\mathrm{b}}$. Whereas the distribution is continuous, 


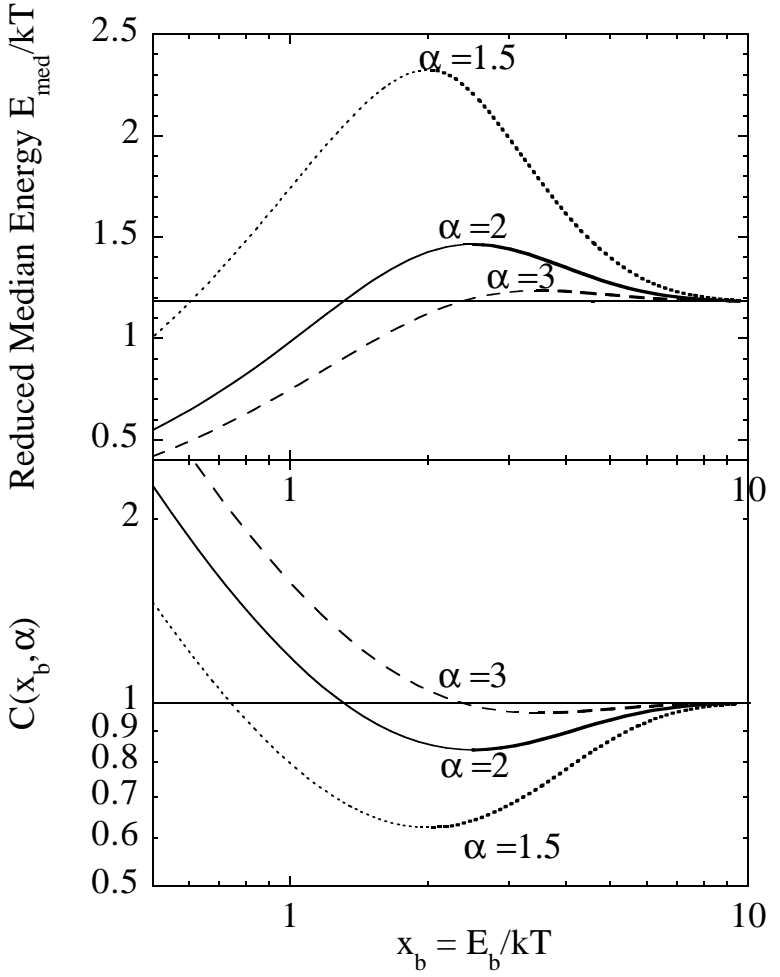

Fig. 2. Variation of the median energy (top panel) and of the normalisation constant (bottom panel) with the break parameter $x_{\mathrm{b}}$. The lines are labeled by the value of the slope parameter $\alpha$. The bold part of the curves corresponds to $x_{\mathrm{b}} \geq \alpha+1 / 2$. The black horizontal lines correspond to the values obtained for a Maxwellian distribution.

its slope changes. The logarithmic slope is $1 / 2-x_{\mathrm{b}}$ on the Maxwellian side and $-\alpha$ on the power-law side. There is no break in the shape of the Hybrid distribution (full line in Fig. 1), only for the critical value of $x_{\mathrm{b}}=\alpha+1 / 2$. For $x_{\mathrm{b}}>\alpha+1 / 2$, the power-law always decreases less rapidly with energy than a Maxwellian distribution and does correspond to an enhanced high energy tail. The contribution of this tail increases with decreasing $x_{\mathrm{b}}$ (and $\alpha$ ). Thus the median energy increases and the normalisation parameter, which scales the Maxwellian part, decreases (see Fig. 2). On the other hand, when $x_{\mathrm{b}}<\alpha+1 / 2$, there is an intermediate region above the energy break where the powerlaw decreases more steeply than a Maxwellian (see dotted line in Fig. 1). This results in a deficit of electrons at these energies as compared to a Maxwellian distribution, more and more pronounced as $x_{\mathrm{b}}$ is small. The median energy thus starts to decrease with decreasing $x_{\mathrm{b}}$ and can be even lower than the median energy of a Maxwellian distribution (Fig. 2). In this paper we only consider the regime where $x_{\mathrm{b}} \geq \alpha+1 / 2$. It corresponds to clear cases where the high energy part of the distribution is indeed increased, as expected when electron are accelerated in shocks. Furthermore, the distribution used here is only an approximation, valid when the hard tail can be considered as a perturbation of the original Maxwellian distribution. The simulations of Bykov \& Uvarov (1999, see their Fig. 2) clearly show that the low energy part of the distribution is less and less well approximated by a Maxwellian distribution, as the "enhanced" high energy tail extends to lower and lower energy (lower "break"). Although we cannot rigorously define a corresponding quantitative lower limit on $x_{\mathrm{b}}$, the cases presented by Bykov \& Uvarov (1999, see their Fig. 2) suggest a limit similar to the one considered here, i.e. a few times the Maxwellian peak energy.

The distribution considered here differs from the socalled "kappa-distribution" or the "power distribution", relevant for other physical conditions (see e.g. Dzifcáková 2000 and references therein). These two distributions have been used to model deviations from a Maxwellian distribution caused by strong plasma inhomogeneities, as in the solar corona, and their impact on the ionization balance has been extensively studied (e.g. Roussel-Dupré 1980; Owocki \& Scudder 1983; Dzifcáková 1992; Dzifcáková 1998). Although the effect of the Hybrid distribution is expected to be qualitatively similar, it has never been quantitatively studied. In the next section we discuss how the ionization and recombination rates are modified, as compared to a pure Maxwellian distribution, depending on the parameters $x_{\mathrm{b}}$ and $\alpha$.

\section{Calculations of the collisional ionization and recombination rates}

Let us consider a collisional process of cross section $\sigma(E)$, varying with energy $E$ of the incident electron. The corresponding rate coefficient $\left(\mathrm{cm}^{3} \mathrm{~s}^{-1}\right)$, either for a Maxwellian distribution or a Hybrid distribution, $f(x)$, is given by:

Rate $=\left(\frac{2 k T}{m_{\mathrm{e}}}\right)^{\frac{1}{2}} \int_{x_{\mathrm{th}}}^{\infty} x^{\frac{1}{2}} \sigma(x k T) f(x) \mathrm{d} x$

with $x_{\mathrm{th}}=E_{\mathrm{th}} / k T . E_{\mathrm{th}}$ corresponds to the threshold energy of the considered process (for $E<E_{\mathrm{th}}, \sigma(E)=0$ ). For the recombination processes, no threshold energy is involved and $x_{\mathrm{th}}=0$.

The rates for the Hybrid distribution depend on $k T$, $x_{\mathrm{b}}$ and $\alpha$ and are noted $C_{\mathrm{I}}^{\mathrm{H}}\left(T, x_{\mathrm{b}}, \alpha\right), \alpha_{\mathrm{RR}}^{\mathrm{H}}\left(T, x_{\mathrm{b}}, \alpha\right)$ and $\alpha_{\mathrm{DR}}^{\mathrm{H}}\left(T, x_{\mathrm{b}}, \alpha\right)$ for the ionization, radiative and dielectronic recombination process respectively. The corresponding rates for the Maxwellian distribution which only depends on $k T$ are $C_{\mathrm{I}}^{\mathrm{M}}(T), \alpha_{\mathrm{RR}}^{\mathrm{M}}(T)$ and $\alpha_{\mathrm{DR}}^{\mathrm{M}}(T)$.

The ionization data are taken from Arnaud \& Rothenflug (1985) and Arnaud \& Raymond (1992), as adopted by Mazzotta et al. (1998) for the most abundant elements considered here. The recombination data are taken from the updated calculations of Mazzotta (1998). In the next sections we outline the general behavior of the rates with the electron distribution parameters, using mostly oxygen ions (but also iron) as illustration.

\subsection{The electronic collisional ionization rates}

The ionization cross sections present a threshold at the first ionization potential of the ionizing ion, $E_{\mathrm{I}}$. The cross sections always present a maximum, at $E_{\mathrm{m}}$, and decrease 


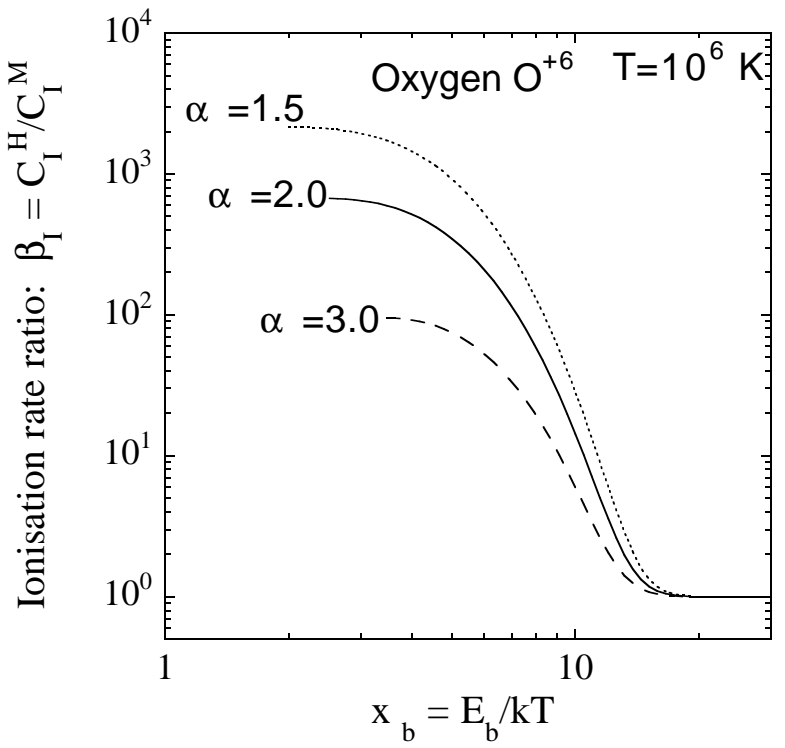

Fig. 3. Variation of the ratio of the ionization rate in a Hybrid distribution over that in a Maxwellian with the same temperature, versus the break parameter $x_{\mathrm{b}}$ of the Hybrid distribution. The curves correspond to different values of the slope parameter $\alpha$ and are labeled accordingly. The ion considered is $\mathrm{O}^{+6}$, the temperature is fixed at $10^{6} \mathrm{~K}$.

as $\ln (E) / E$ at very high energies (e.g., Tawara et al. 1985). The ionization rate is very sensitive to the proportion of electrons above the threshold and the modification of the ionization rate for the Hybrid distribution depends on how the high energy tail affects this proportion.

Parametric formulae for the ionization cross sections are available from the litterature and it is easy to derive the corresponding rates for the Hybrid distribution. This is detailed in Appendix A.

To understand the influence of the presence of a high energy power-law tail in the electron distribution, we computed the ratio $\beta_{\mathrm{I}}\left(T, x_{\mathrm{b}}, \alpha\right)=C_{\mathrm{I}}^{\mathrm{H}}\left(T, x_{\mathrm{b}}, \alpha\right) / C_{\mathrm{I}}^{\mathrm{M}}(T)$, of the ionization rate in a Hybrid distribution over that in a Maxwellian with the same temperature. This ratio is plotted in Figs. 3 to 7 for different ions and values of the parameters $x_{\mathrm{b}}$ and $\alpha$.

Let us first consider $\mathrm{O}^{+6}$. Its ionization potential is $E_{\mathrm{I}}=739 \mathrm{eV}$ and the cross section is maximum at about $3 E_{\mathrm{I}}$. Its abundance, for a Maxwellian electron distribution, is maximum at $T^{*} \simeq 10^{6} \mathrm{~K}$ under ionization equilibrium (Arnaud \& Rothenflug 1985). At this temperature, the threshold energy is well above the thermal energy $\left(E_{\mathrm{I}} / k T \sim 8\right)$ and only the very high energy tail of the Maxwellian contributes to $C_{\mathrm{I}}^{\mathrm{M}}(T)$, i.e. a small fraction of the electron distribution. This fraction is dramatically increased in the Hybrid distribution as soon at the break energy is not too far off from the threshold, $x_{\mathrm{b}} \sim 15$ for $\mathrm{O}^{+6}$ (Fig. 3). The enhancement factor $\beta_{\mathrm{I}}\left(T, x_{\mathrm{b}}, \alpha\right)$ naturally increases with decreasing break $x_{\mathrm{b}}$ and slope $\alpha$ parameters (Fig. 3), since the distribution median energy increases when these parameters are decreased (Fig. 2).

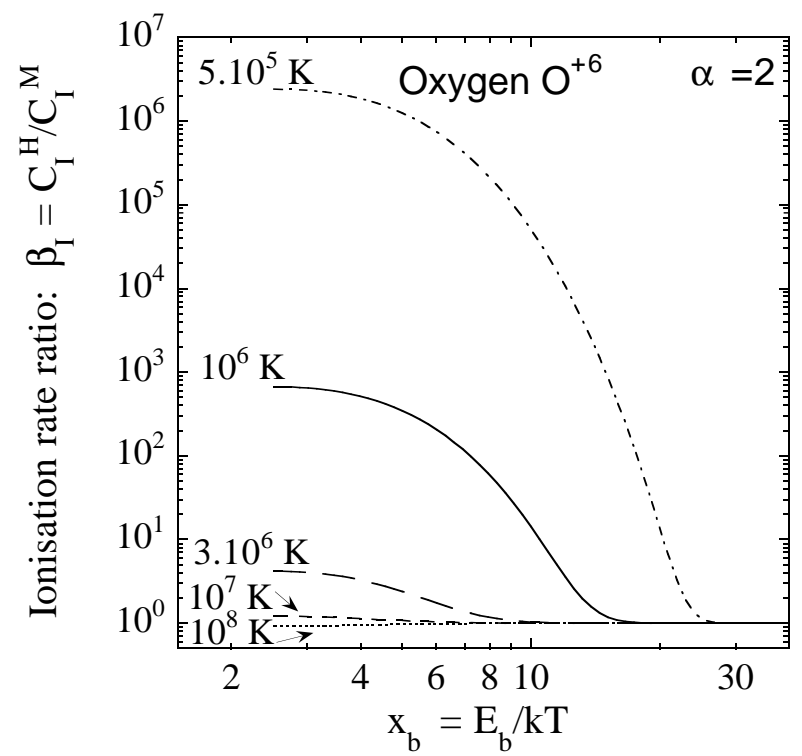

Fig. 4. Same as Fig. 3 but the parameter $\alpha$ is now fixed to 2 . The curves correspond to different values of the temperature.

This behavior versus $x_{\mathrm{b}}$ and $\alpha$ is general at all temperatures as illustrated in Fig. 4, provided that the thermal energy is not too close to $E_{\mathrm{m}}$, i.e. that the majority of the contribution to the ionization rate is from electrons with energies corresponding to the increasing part of the ionization cross section. If this is no more the case, the ionization rate starts to decrease with increasing distribution median energy. Thus, for high enough values of the temperature (see the curve at $T=10^{8} \mathrm{~K}$ in Fig. 4), the factor $\beta_{\mathrm{I}}\left(T, x_{\mathrm{b}}, \alpha\right)$ becomes less than unity and decreases with decreasing $x_{\mathrm{b}}$. The correction factor is small (around $\sim 10 \%$ ) however in that case.

More generally the enhancement factor $\beta_{\mathrm{I}}\left(T, x_{\mathrm{b}}, \alpha\right)$ at fixed values of $x_{\mathrm{b}}$ and $\alpha$, depends on the temperature (Fig. 4). It decreases with increasing temperature: the peak of the distribution is shifted to higher energy as the ratio $k T / E_{\mathrm{I}}$ increases and the enhancement due to the contribution of the hard energy tail decreases.

The qualitative behavior outlined above does not depend on the ion considered. We plotted in Figs. 5 and 6 the enhancement factor for the different ions of oxygen and a choice of iron ions at $T^{*}$ (the temperature of maximum ionization fraction of the ion for a Maxwellian electron distribution under ionization equilibrium). $E_{\mathrm{I}} / k T^{*}$ is always greater than unity and the ionization rates are increased by the Hybrid distribution, the enhancement factor $\beta_{\mathrm{I}}\left(T, x_{\mathrm{b}}, \alpha\right)$ increasing with decreasing $x_{\mathrm{b}}$. However this enhancement factor differs from ion to ion, it generally increases with increasing $E_{\mathrm{I}} / k T^{*}$ value (approximatively with an exponential dependence), as shown in Fig. 7. This is again due to the relative position of the peak of the distribution with respect to the threshold energy. Note that $E_{\mathrm{I}} / k T^{*}$ is generally smaller for more ionized ions (but this is not strictly true) so that low charge species are generally more affected by the Hybrid distribution. 


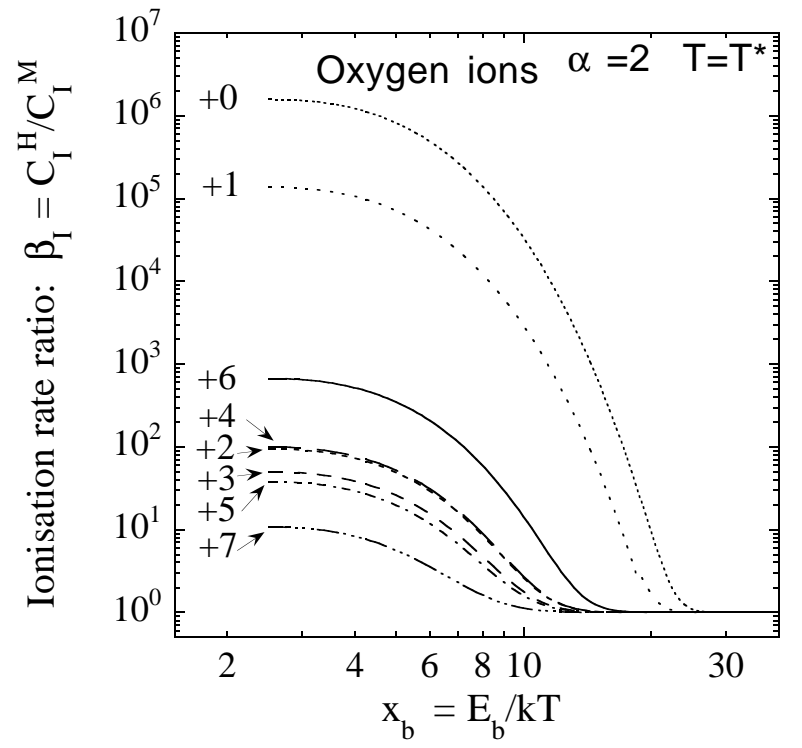

Fig. 5. Same as Fig. 3 for the different ions of oxygen. Each curve is labeled by the charge of the ion considered. The slope parameter is fixed to $\alpha=2$ and the temperature for each ion is fixed at the value, $T^{*}$, where the abundance of the ion is maximum for a Maxwellian electron distribution.

In summary, the Hybrid rates are increased with respect to the Maxwellian rates except at very high temperature. The enhancement factor depends on the temperature, mostly via the factor $E_{\mathrm{I}} / k T$. It increases dramatically with decreasing temperature and is always important at $T^{*}$, where it can reach several orders of magnitude. The ionization balance is thus likely to be affected significantly, whereas the effect should be smaller in ionizing plasmas but important in recombining plasmas. For $x_{\mathrm{b}}$ typically lower than 10-20 (with this upper limit higher for lower temperature, see Fig. 4), the impact of the Hybrid rate increases with decreasing $x_{\mathrm{b}}$ and $\alpha$.

The ionization rates for a Hybrid distribution are less dependent on the temperature than the Maxwellian rates, as illustrated in Figs. 8 and 9. This is a direct consequence of the temperature dependance of the enhancement factor: as this factor increases with decreasing temperature, the Hybrid ionization rate decreases less steeply with temperature than the Maxwellian rates. More precisely, as derived from the respective expression of the rates at low temperature (respectively Eqs. (A.2) and (A.10)), the Maxwellian rate falls off exponentially (as $\mathrm{e}^{-E_{\mathrm{I}} / k T}$ ) with decreasing temperature, whereas the Hybrid rate only decreases as a power-law. As expected, one also notes that the modification of the rates is more pronounced for lower value of $x_{\mathrm{b}}$ (compare the two figures corresponding to $x_{\mathrm{b}}=10$ and $\left.x_{\mathrm{b}}=2.5\right)$.

\subsection{The recombination rates}

\subsubsection{The radiative recombination rates}

The radiative recombination rates are expected to be less affected by the Hybrid distribution, since the cross sections for recombination decrease with energy and no threshold exists. As the net effect of the high energy tail

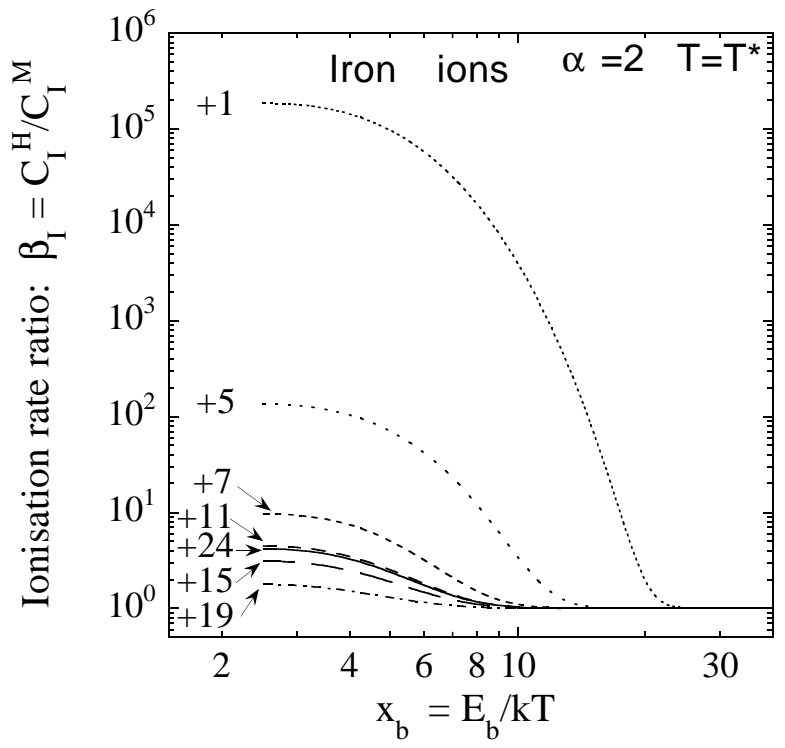

Fig. 6. Same as Fig. 5 for the different ions of iron.

present in the hybrid distribution is to increase the median energy of the distribution (cf. Fig. 2), as compared to a Maxwellian, the radiative recombination rates are decreased.

To estimate the corresponding dumping factor, $\beta_{\mathrm{RR}}\left(x_{\mathrm{b}}, \alpha\right)=\alpha_{\mathrm{RR}}^{\mathrm{H}}\left(T, x_{\mathrm{b}}, \alpha\right) / \alpha_{\mathrm{RR}}^{\mathrm{M}}(T)$, we follow the method used by Owocki \& Scudder (1983). We assume that the radiative recombination cross section varies as a power-law in energy:

$\sigma_{\mathrm{RR}}(E) \propto E^{-a}$

which corresponds to a recombination rate (Eq. (8)), for a Maxwellian distribution (Eq. (4)), varying as:

$\alpha_{\mathrm{RR}}^{\mathrm{M}}(T) \propto T^{\eta}$

with $\eta=a-\frac{1}{2}$.

The dumping factor computed for such a power-law cross section (Eqs. (8) with (9)) is:

$\beta_{\mathrm{RR}}\left(x_{\mathrm{b}}, \alpha\right)=\frac{\int_{0}^{\infty} x^{-\eta} f^{\mathrm{H}}(x) \mathrm{d} x}{\int_{0}^{\infty} x^{-\eta} f^{\mathrm{M}}(x) \mathrm{d} x}$.

Note that the dumping factor is independent of the temperature. It depends on the ion considered via the $\eta$ parameter. Replacing the Maxwellian and Hybrid distribution functions by their expression (respectively Eqs. (4) and (6)) we obtain:

$\beta_{\mathrm{RR}}\left(x_{\mathrm{b}}, \alpha\right)=\frac{C\left(x_{\mathrm{b}}, \alpha\right)}{\Gamma\left(\frac{3}{2}-\eta\right)}\left[\gamma\left(\frac{3}{2}-\eta, x_{\mathrm{b}}\right)+\frac{x_{\mathrm{b}}^{\frac{3}{2}-\eta} \mathrm{e}^{-x_{\mathrm{b}}}}{\alpha+\eta-1}\right]$

This estimate of the dumping factor is only an approximation, since the radiative recombination has to be computed by summing over the various possible states of the recombined ions, taking into account the respective different cross sections. Furthermore, even if often the radiative recombination rate can be approximated by a power-law 


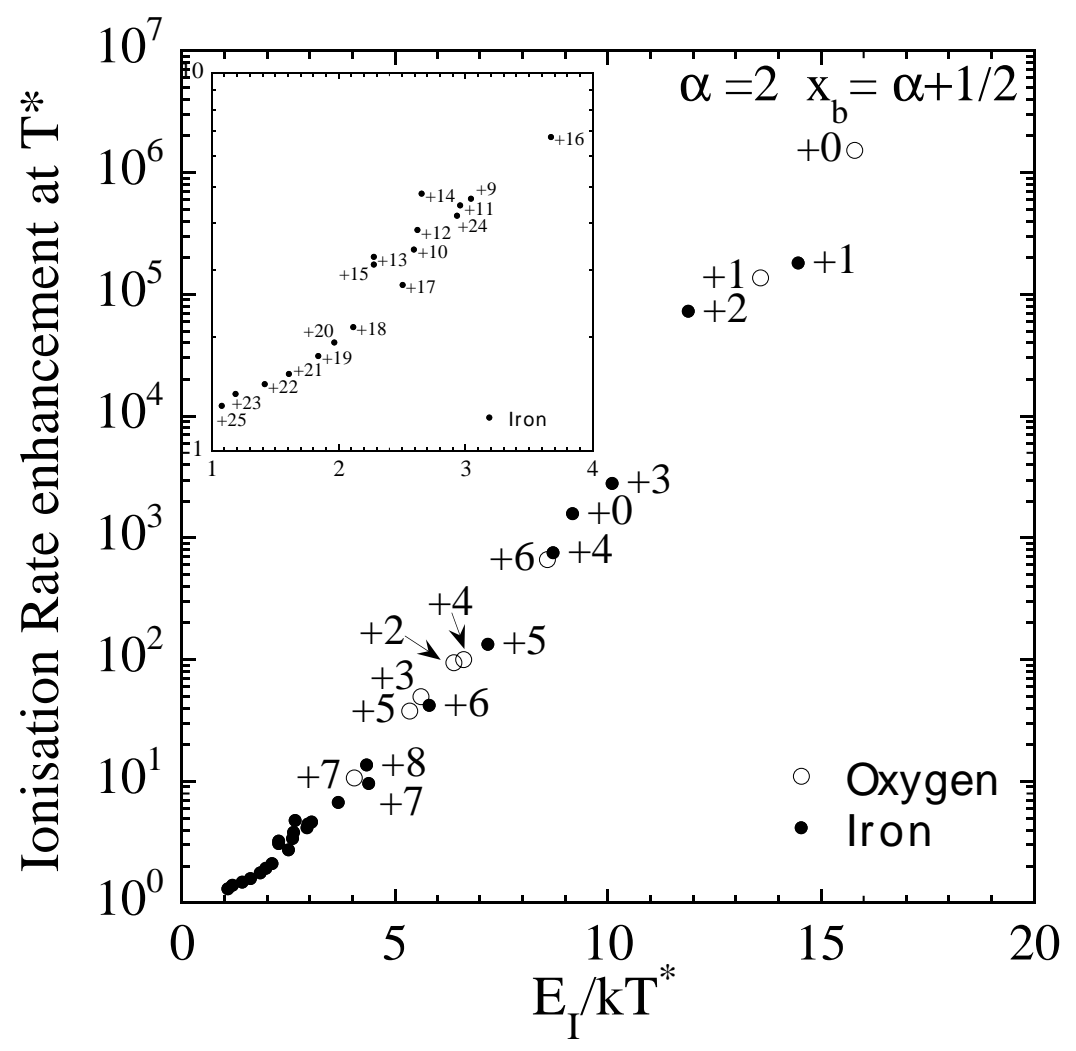

Fig. 7. Maximum enhancement ratio of the ionization rate, $\beta_{\mathrm{I}}\left(T, x_{\mathrm{b}}, \alpha\right)$, at $T^{*}$ versus $E_{\mathrm{I}} / k T^{*}$. $T^{*}$ is the temperature at which the abundance of the ion is maximum for a Maxwellian electron distribution and $E_{\mathrm{I}}$ is the first ionization potential of the ion. Each point corresponds to an ion of oxygen (open circle) or iron (filled circles). The points are labeled by the charge of the corresponding ion. The slope parameter is fixed to $\alpha=2$ and the break is $x_{\mathrm{b}}=\alpha+\frac{1}{2}$, corresponding to a maximum enhancement of the ionization rate.

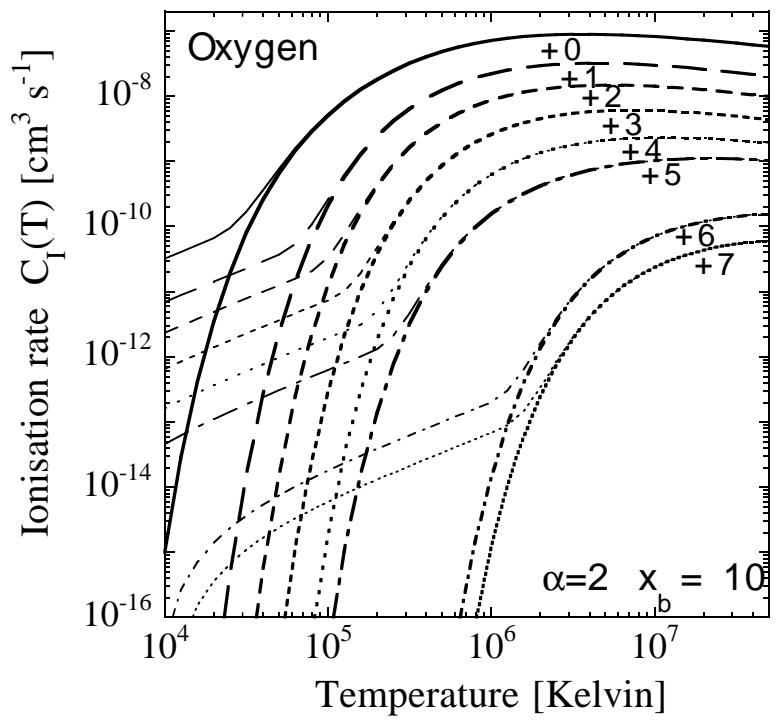

Fig. 8. Variation of the ionization rate with temperature. Each curve corresponds to an ion of oxygen and is labeled accordingly. Black lines: rates for a Hybrid electron distribution with $\alpha=2$ and $x_{\mathrm{b}}=10$. Black thick lines: rates for a Maxwellian distribution.

in a given temperature range, this does not mean that the underlying cross section is well approximated by a unique power-law.

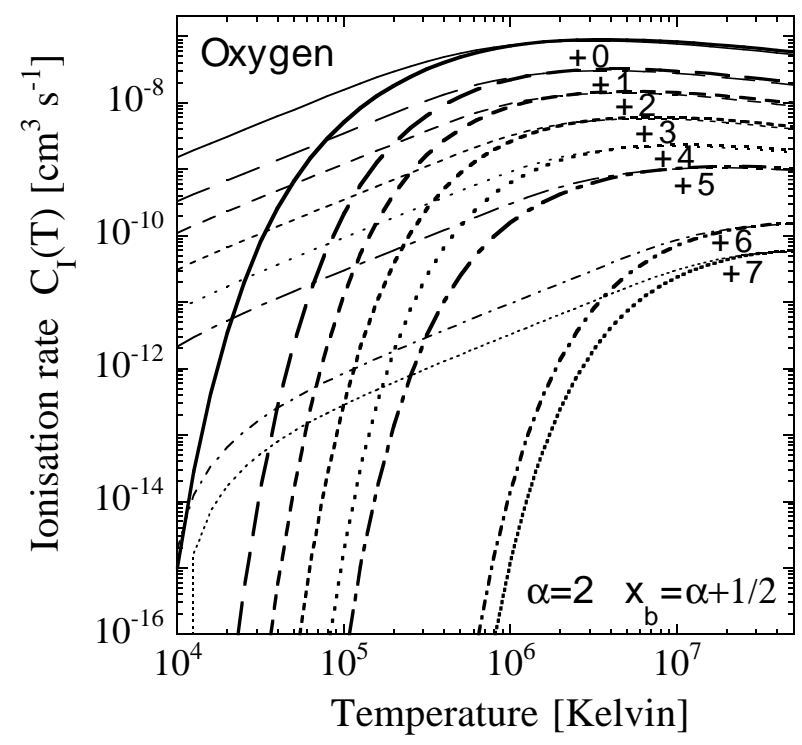

Fig. 9. Same as Fig. 8 for $\alpha=2$ and $x_{\mathrm{b}}=\alpha+\frac{1}{2}=2.5$.

However as we will see the correction factor is small, and we can reasonably assume that it allows a fair estimate of the true Hybrid radiative recombination rates. To minimize the errors, the Hybrid radiative recombination rate has to be calculated from the best estimate of the 


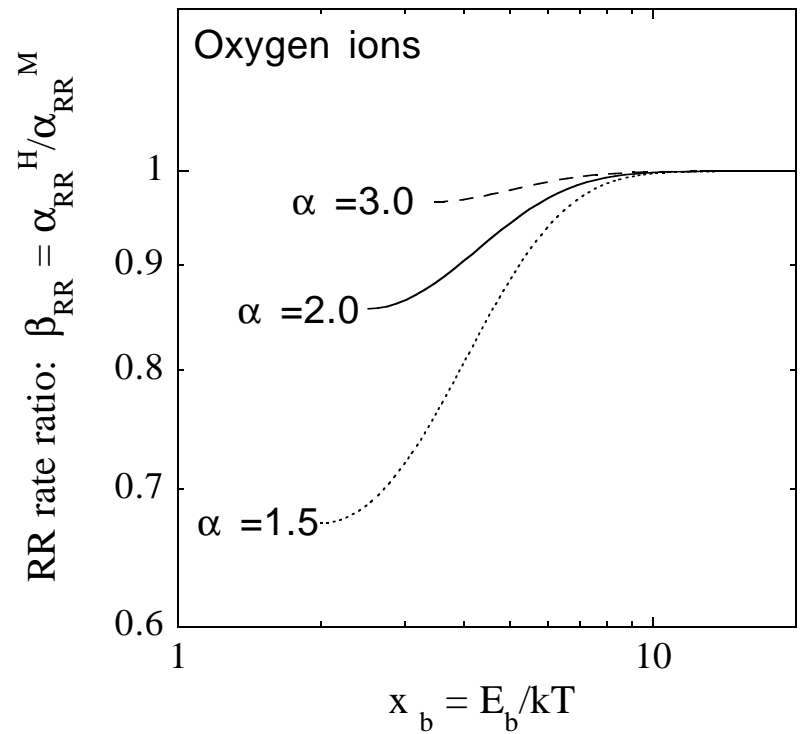

Fig. 10. Dumping factor for the radiative recombination rates, $\beta_{\mathrm{RR}}\left(x_{\mathrm{b}}, \alpha\right)$, of oxygen ions versus the break parameter $x_{\mathrm{b}}$. The curves correspond to different values of the slope parameter $\alpha$ and are labeled accordingly. This dumping factor is independent of temperature.

Maxwellian rates, multiplied by this approximation of the dumping factor:

$\alpha_{\mathrm{RR}}^{\mathrm{H}}\left(T, x_{\mathrm{b}}, \alpha\right)=\beta_{\mathrm{RR}}\left(x_{\mathrm{b}}, \alpha\right) \alpha_{\mathrm{RR}}^{\mathrm{M}}(T)$

where $\alpha_{\mathrm{RR}}^{\mathrm{M}}(T)$ is as given in Mazzotta et al. (1998).

The parameters $\eta$ for the various ions are taken from Aldrovandi \& Péquignot (1973), when available. For other ions we used a mean value of $\eta=0.8$ corresponding to the mean value $\langle\eta\rangle$ reported in Arnaud \& Rothenflug (1985). The exact value has a negligible effect on the estimation of the radiative recombination rates.

The dumping factor is plotted in Fig. 10 for the various ions of oxygen. In that case a common $\eta$ value is used. The dumping factor decreases with decreasing values of $x_{\mathrm{b}}$ and $\alpha$, following the increase of the distribution median energy. The modification is however always modest, at most $15 \%$ for $\alpha=2$. For iron, plotted in Fig. 11 for $\alpha=2$, the value of $\eta$ slightly changes with the considered ions, but this only yields negligible variations in the dumping factor.

\subsubsection{The dielectronic recombination rates}

The dielectronic recombination is a resonant process involving bound states at discrete energies $E_{i}$ and the rates have to be computed by summing the contribution of many such bound states. According to Arnaud \& Raymond (1992), and Mazzotta et al. (1998), the dielectronic recombination rates for a Maxwellian distribution can be fitted accurately by the formula:

$\alpha_{\mathrm{DR}}^{\mathrm{M}}(T)=T_{\mathrm{eV}}^{-3 / 2} \sum_{i} c_{i} \mathrm{e}^{-x_{i}} \quad \mathrm{~cm}^{3} \mathrm{~s}^{-1}$

where $T_{\mathrm{eV}}$ is the temperature expressed in $\mathrm{eV}$ and $x_{i}=$ $E_{i} / k T$. The numerical values for $c_{i}$ and $E_{i}$ are taken from

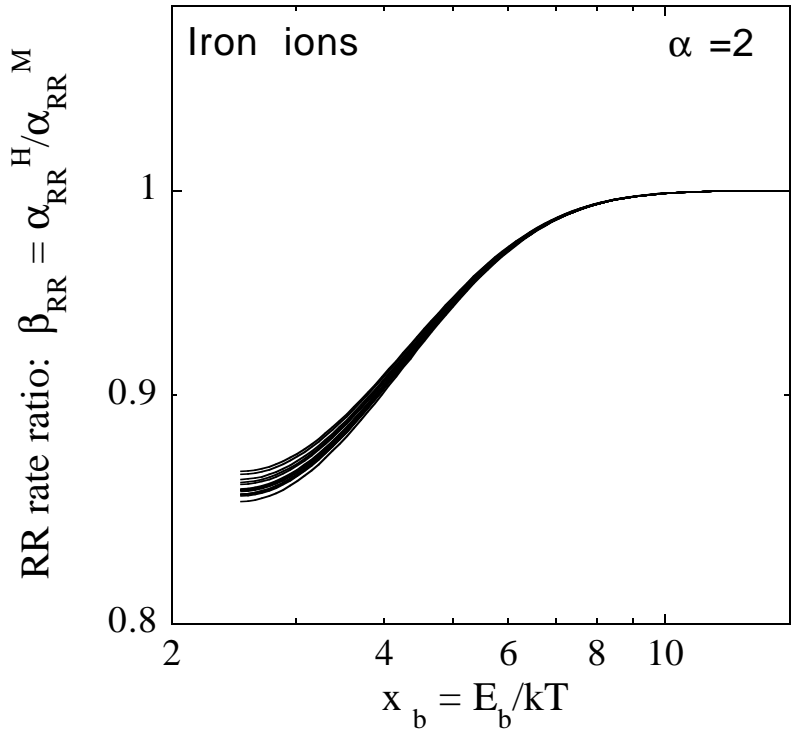

Fig. 11. Same as Fig. 10 for iron ions and $\alpha=2$. The slight variations among the species are due to the corresponding variations in the adopted $\eta$ values (see Eq. (12)).

Mazzotta et al. (1998). Only a few terms (typically 1 to 4 ) are introduced in this fitting formula. They roughly correspond to the dominant transitions for the temperature range considered.

Following again the method used by Owocki \& Scudder (1983), we thus assume that the corresponding dielectronic recombination cross section can be approximated by:

$\sigma_{\mathrm{DR}}(E)=\sum_{i} C_{i} \delta\left(E-E_{i}\right)$ with $C_{i}=\frac{c_{i}\left(2 \pi m_{\mathrm{e}}\right)^{\frac{1}{2}}}{4 E_{i}}$

The relation between $C_{i}$ and $c_{i}$ is obtained by comparing Eq. (14) with the equation obtained by integrating (Eq. (8)) the above cross section over a Maxwellian distribution (Eq. (4)). The dielectronic rates can then be computed from Eq. (8), with the cross section given by Eq. (15) and the distribution function given by Eq. (6):

$$
\begin{aligned}
\alpha_{\mathrm{DR}}^{\mathrm{H}}\left(T, x_{\mathrm{b}}, \alpha\right)= & C\left(x_{\mathrm{b}}, \alpha\right) T_{\mathrm{eV}}^{-3 / 2} \sum_{i, x_{i} \leq x_{\mathrm{b}}} c_{i} \mathrm{e}^{-x_{\mathrm{i}}}+C\left(x_{\mathrm{b}}, \alpha\right) \\
& \times T_{\mathrm{eV}}^{-3 / 2} \mathrm{e}^{-x_{\mathrm{b}}} \sum_{i, x_{i}>x_{\mathrm{b}}} c_{i}\left(\frac{x_{i}}{x_{\mathrm{b}}}\right)^{-\alpha-\frac{1}{2}} \cdot
\end{aligned}
$$

Note that this estimate of $\alpha_{\mathrm{DR}}^{\mathrm{H}}\left(T, x_{\mathrm{b}}, \alpha\right)$ is only an approximation, for the same reasons outlined above for the radiative recombination rates.

To understand the effect of the hybrid distribution, let us assume that only a single energy $E_{\mathrm{DR}}$ is dominant, corresponding to a simple Dirac cross section at this energy. In that case, from Eq. (8), the ratio of the dielectronic recombination rate in a Hybrid distribution over that in a Maxwellian with the same temperature, $\beta_{\mathrm{DR}}\left(T, x_{\mathrm{b}}, \alpha\right)=\alpha_{\mathrm{DR}}^{\mathrm{H}}\left(T, x_{\mathrm{b}}, \alpha\right) / \alpha_{\mathrm{DR}}^{\mathrm{M}}(T)$, is simply the ratio of the Hybrid to the Maxwellian function at the resonance 


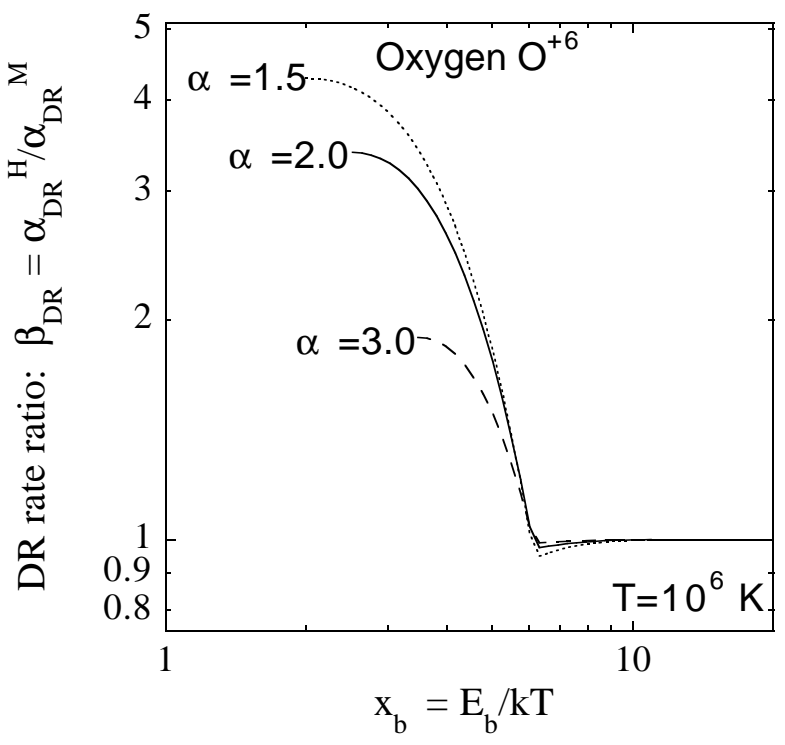

Fig. 12. Variation of the ratio of the DR rate in a Hybrid distribution over that in a Maxwellian with the same temperature, versus the break parameter $x_{\mathrm{b}}$ of the Hybrid distribution. The curves correspond to different values of the slope parameter $\alpha$ and are labeled accordingly. The ion considered is $\mathrm{O}^{+6}$, the temperature is fixed at $10^{6} \mathrm{~K}$.

energy. Its expression depends on the position of the resonance energy with respect to the energy break. In reduced energy coordinates, we obtain from Eqs. (4) and (6):

$$
\begin{gathered}
\beta_{\mathrm{DR}}\left(T, x_{\mathrm{b}}, \alpha\right)=C\left(x_{\mathrm{b}}, \alpha\right) \quad \text { for } \frac{E_{\mathrm{DR}}}{k T} \leq x_{\mathrm{b}} \\
\beta_{\mathrm{DR}}\left(T, x_{\mathrm{b}}, \alpha\right)=C\left(x_{\mathrm{b}}, \alpha\right) \mathrm{e}^{\left(\frac{E_{\mathrm{DR}}}{k T}-x_{\mathrm{b}}\right)}\left(\frac{E_{\mathrm{DR}}}{x_{\mathrm{b}} k T}\right)^{-\left(\frac{1}{2}+\alpha\right)} \\
\text { for } \frac{E_{\mathrm{DR}}}{k T} \geq x_{\mathrm{b}}
\end{gathered}
$$

For $\left(x_{\mathrm{b}} k T\right)>E_{\mathrm{DR}}$ (i.e. at high temperature or high value of $x_{\mathrm{b}}$ ), the resonance lies in the Maxwellian part of the distribution. $\beta_{\mathrm{DR}}\left(T, x_{\mathrm{b}}, \alpha\right)$ is independent of the temperature and the dielectronic recombination rates are decreased, following the variation of the normalisation factor, $C\left(x_{\mathrm{b}}, \alpha\right)$, i.e. the decrease is modest (see Fig. 2).

For $\left(x_{\mathrm{b}} k T\right)<E_{\mathrm{DR}}$ the resonance lies in the power-law part of the distribution. The increase of the dielectronic recombination rate can be dramatic, increasing with decreasing $x_{\mathrm{b}}$ and $\alpha$.

These effects of the Hybrid distribution on the dielectronic recombination rates are illustrated in Figs. 12 to 15, where we plotted the factor $\beta_{\mathrm{DR}}\left(T, x_{\mathrm{b}}, \alpha\right)$ for various ions and values of the parameters. The factors are computed exactly from Eqs. (14) and (16).

In Fig. 12 we consider $\mathrm{O}^{+6}$ at the temperature of its maximum ionization fraction, $T^{*}=10^{6} \mathrm{~K}$. For this ion only one term is included in the rate estimate, with $E_{\mathrm{DR}}=529 \mathrm{eV}$, and $E_{\mathrm{DR}} / k T=6.1$ at the temperature considered. We plotted the variation of $\beta_{\mathrm{DR}}\left(T, x_{\mathrm{b}}, \alpha\right)$ with $x_{\mathrm{b}}$ for $\alpha=3, \alpha=2$ and $\alpha=1.5$. For $x_{\mathrm{b}}>6.1$ the

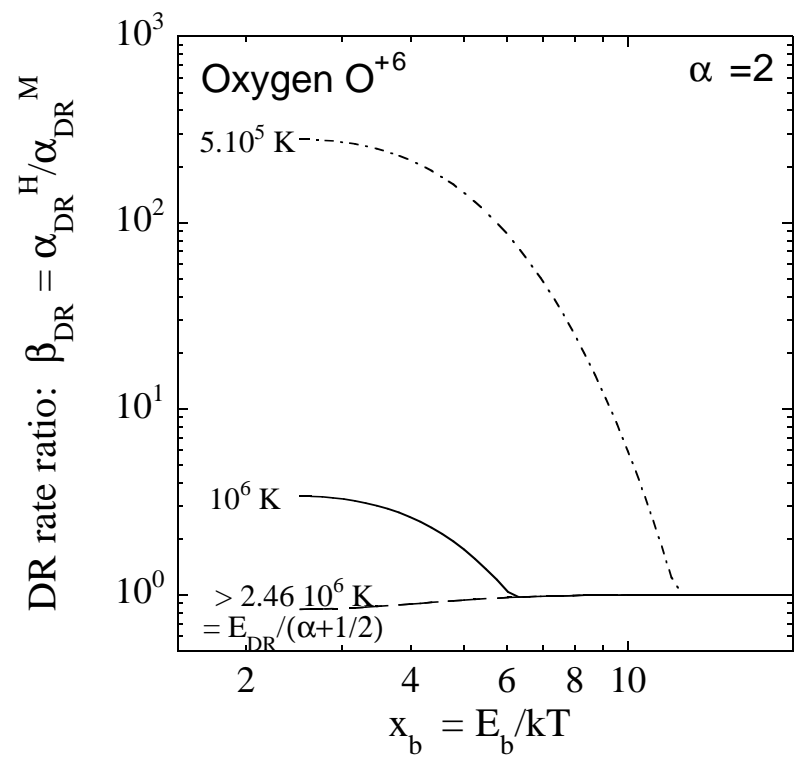

Fig. 13. Same as Fig. 12 but the parameter $\alpha$ is now fixed to 2 . The curves correspond to different values of the temperature and are labelled accordingly.

"resonance" energy $E_{\mathrm{DR}}$ lies in the Maxwellian part of the distribution and the dielectronic recombination rate is decreased as compared to a Maxwellian, but by less than $10 \%$, following the variation of the normalisation factor $C\left(x_{\mathrm{b}}, \alpha\right)$. For smaller values of $x_{\mathrm{b}}$, the rate is increased significantly, up to a factor of 5 for $\alpha=1.5$.

We consider other temperatures, fixing $\alpha$ to $\alpha=2$, in Fig. 13. Since we only consider the parameter range $x_{\mathrm{b}}>\alpha+1 / 2$, there is a threshold temperature, $k T>$ $E_{\mathrm{DR}} /(\alpha+1 / 2)$, above which the resonance always falls in the Maxwellian part. The dielectronic recombination rate is decreased via the factor $C\left(x_{\mathrm{b}}, \alpha\right)$. This factor slightly decreases with decreasing $x_{\mathrm{b}}$ (cf. Fig. 2). At lower temperature, the resonance energy can fall above the break, provided that $x_{\mathrm{b}}$ is small enough $\left(x_{\mathrm{b}}<E_{\mathrm{DR}} / k T\right)$. This occurs at smaller $x_{\mathrm{b}}$ for higher temperature and the enhancement at a given $x_{\mathrm{b}}$ increases with decreasing temperature.

We display in Figs. 14 and 15 the variation of the factor $\beta_{\mathrm{DR}}\left(T, x_{\mathrm{b}}, \alpha\right)$ with $x_{\mathrm{b}}$ (for $\alpha=2$ ), for the different ions of oxygen and iron, at the temperature of maximum ionization fraction for a Maxwellian distribution under ionization equilibrium. For most of the ions this temperature is above the threshold temperature, $k T=E_{\mathrm{DR}} /(\alpha+1 / 2)$, for all the resonances and the dielectronic rate is decreased. For the ions for which this is not the case $\left(\mathrm{O}^{+1}, \mathrm{O}^{+6}\right.$ and from $\mathrm{Fe}^{+1}$ to $\mathrm{Fe}^{+5}$ ), the dielectronic rate can be increased significantly (by a factor between 2 to 5 ) provided $x_{\mathrm{b}}$ is small enough (typically $x_{\mathrm{b}}=2.5-5$ ). The increase starts as soon as $x_{\mathrm{b}}<E_{\mathrm{DR}} / k T^{*}$ for the oxygen ions. The behavior of $\beta_{\mathrm{DR}}\left(T, x_{\mathrm{b}}, \alpha\right)$ is more complex for the iron ions (two breaks in the variation of $\left.\beta_{\mathrm{DR}}\left(T, x_{\mathrm{b}}, \alpha\right)\right)$, due to the presence of more than one dominant resonance energy (more than one term), taken into account in the computation of the dielectronic rate. 


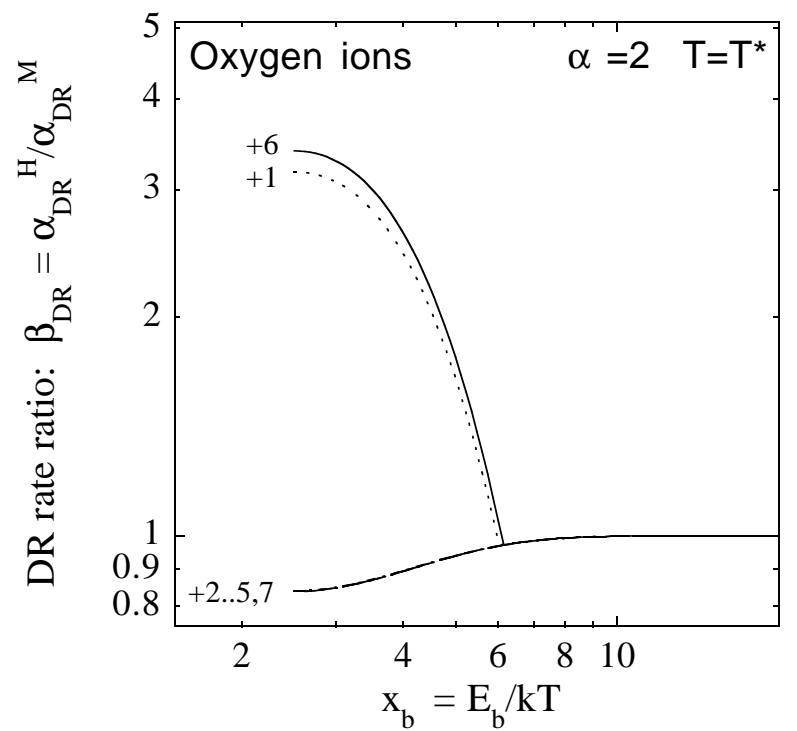

Fig. 14. Same as Fig. 12 for the different ions of oxygen. Each curve is labeled by the charge of the ion considered. The slope parameter is fixed to $\alpha=2$ and the temperature for each ion is fixed at the value, $T^{*}$, where the abundance of the ion is maximum for a Maxwellian electron distribution.

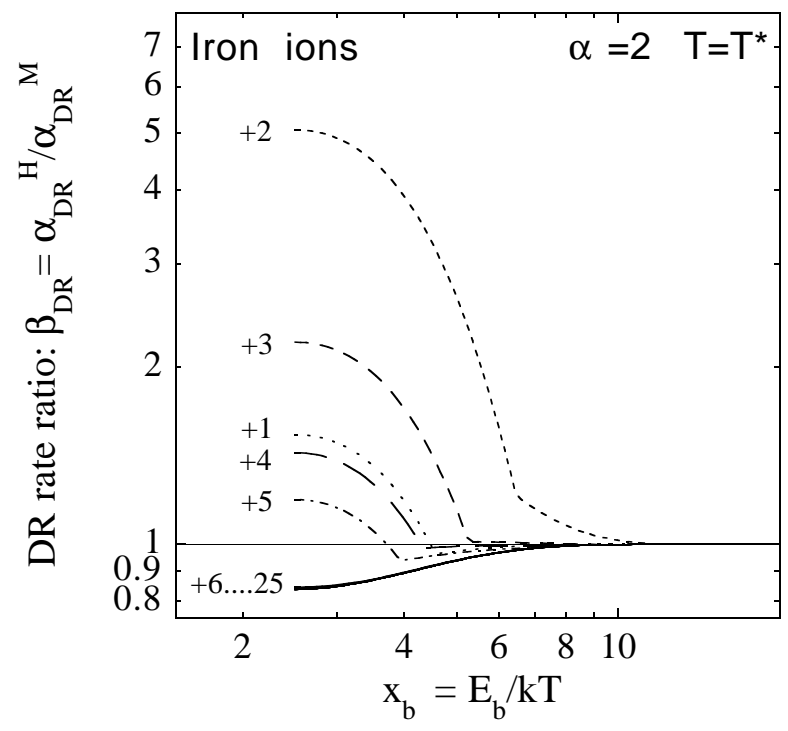

Fig. 15. Same as Fig. 14 for iron.

In conclusion, the effect of the hybrid distribution on the dielectronic rate depends on the position of the resonance energy as compared to the power-law energy break. It can only be increased if $k T<E_{\mathrm{DR}} /(\alpha+1 / 2)$. At high temperature, the dielectronic recombination rate is slightly decreased.

\subsubsection{The total recombination rates}

At $x_{\mathrm{b}}=10$, the total rates are basically unchanged by the Hybrid distribution. For $x_{\mathrm{b}}=2.5=\alpha+1 / 2$ (Fig. 16), the total rates are more significantly changed. The radiative recombination rate increases with decreasing temperature and it usually dominates the total recombination rate in

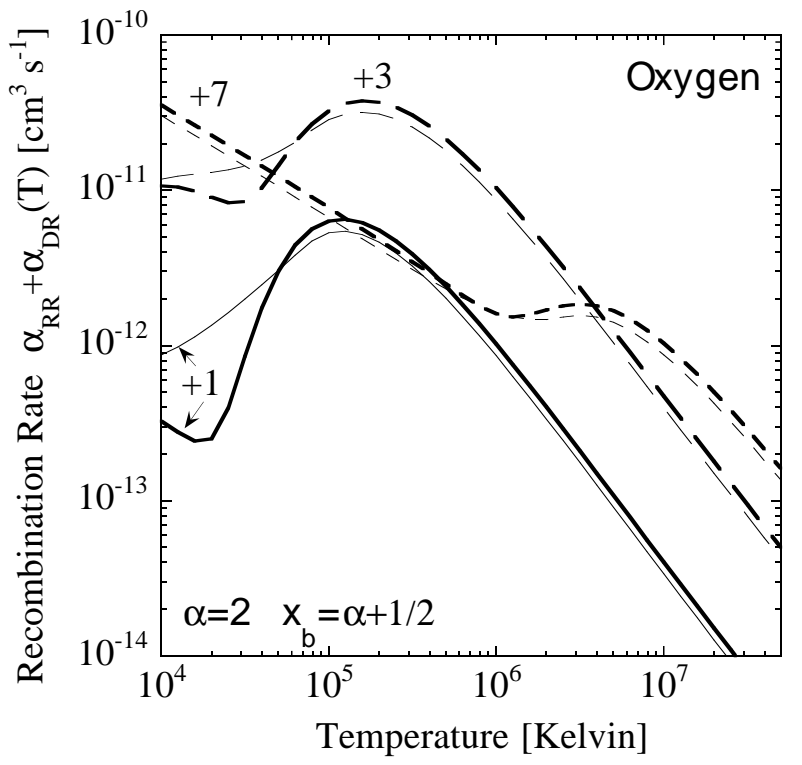

Fig. 16. Variation of the total recombination rate with temperature. Each curve corresponds to an ion and is labeled accordingly. Black lines: rates for a Hybrid electron distribution with $\alpha=2$ and $x_{\mathrm{b}}=\alpha+\frac{1}{2}=2.5$. Black thick lines: rates for Maxwellian distribution.

the low temperature range. As the dielectronic rate is increased by the Hybrid distribution only at low temperature, there are very few ions for which the total recombination rate can be actually increased. This only occurs in a small temperature range, in the rising part of the dielectronic rate. One also notes the expected slight decrease of the radiative recombination rates (when it is dominant at low temperature) and of the dielectronic rate at high temperature.

\section{Ionization equilibria}

\subsection{Collisional ionization equilibrium (CIE)}

The ionization equilibrium fractions, for coronal plasmas, can be computed from the rates described in the previous sections. In the low density regime (coronal plasmas) the steady state ionic fractions do not depend on the electron density and the population density ratio $N_{Z, z+1} / N_{Z, z}$ of two adjacent ionization stages $Z^{+(z+1)}$ and $Z^{+z}$ of element $Z$ can be expressed by:

$\frac{N_{Z, z+1}}{N_{Z, z}}=\frac{C_{I}^{Z, z}}{\alpha_{R}^{Z, z+1}}$

where $C_{I}^{Z, z}$ and $\alpha_{R}^{Z, z+1}$ are the ionization and total recombination rates of ion $Z^{+z}$ and $Z^{+(z+1)}$ respectively. To assess the impact of the Hybrid rates on the ionization balance, we computed the variation with temperature of the mean electric charge of the plasma. This variation is compared with the variation obtained for a Maxwellian electron distribution in Fig. 17 for oxygen and iron and for different values of the parameters $x_{\mathrm{b}}$ and $\alpha$. 

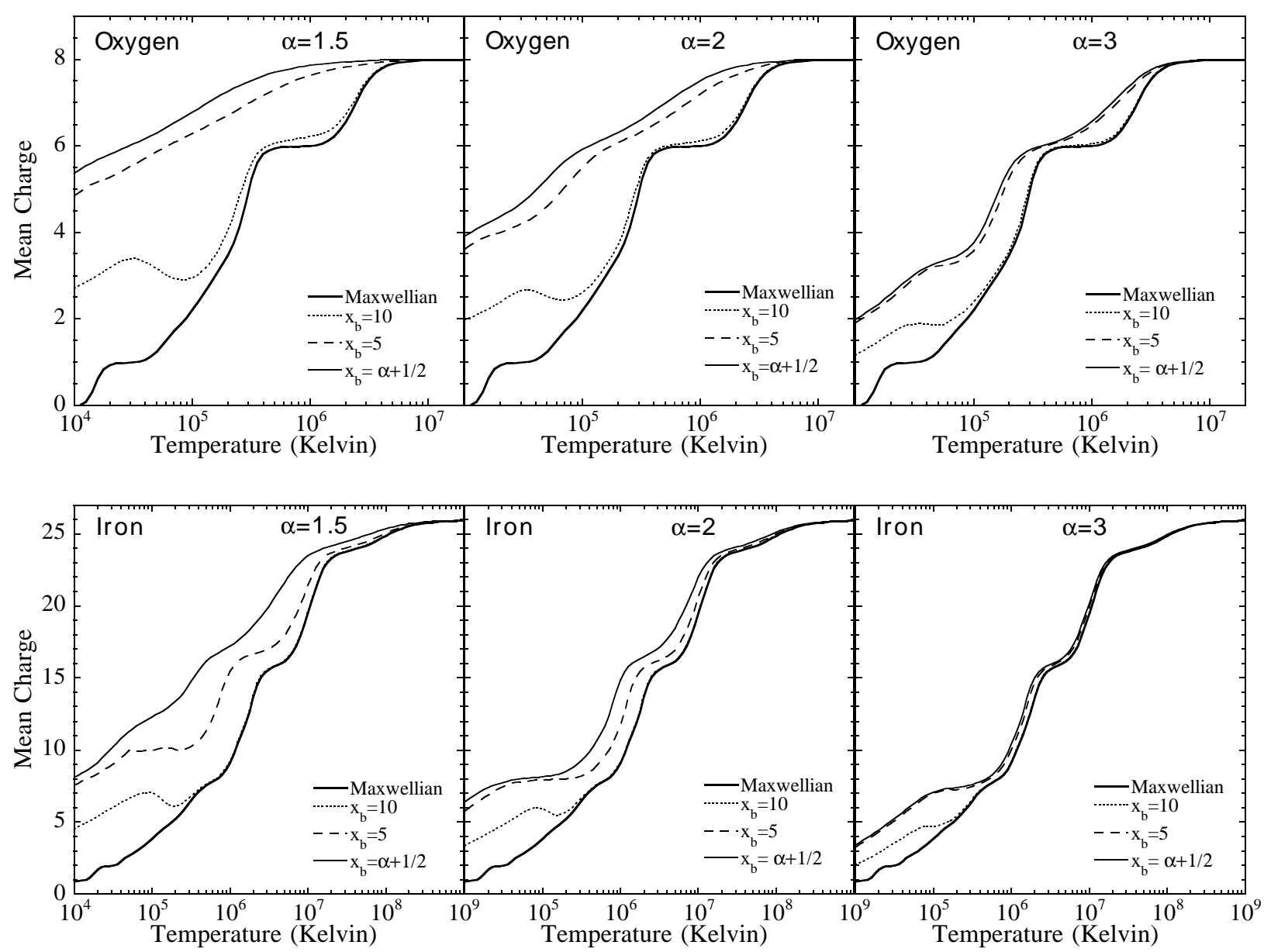

Fig. 17. Mean electric charge versus temperature for oxygen (top panels) and iron (bottom panels) for different electron distributions. Black thick lines: Maxwellian electron distribution. Black thin lines: Hybrid distribution with $\alpha=1.5$ (left panels), $\alpha=2$ (middle panels) and $\alpha=3$ (right panels). Three values of $x_{\mathrm{b}}$ are considered: $x_{\mathrm{b}}=10$ (dotted line), $x_{\mathrm{b}}=5$ (dashed line) and the extreme value $x_{\mathrm{b}}=\alpha+1 / 2$ (full line).

As expected, the plasma is always more ionized for a Hybrid electron distribution than for a Maxwellian distribution. The mean charge at a given temperature is increased, since the enhancement of the ionization rate is always much more important than a potential increase of the dielectronic rate (e.g. compare Figs. 5 and 14). The effect of the Hybrid distribution on the plasma ionization state is thus governed by the enhancement of the ionization rates. The enhancement of the plasma mean charge is more pronounced for smaller values of $x_{\mathrm{b}}$ and smaller values of $\alpha$ (Fig. 17), following the same behavior observed for the ionization rates (due to the increasing influence of the high energy tail). Similarly the effect is more important at low temperature, and a clear signature of the Hybrid distribution is the disappearance of the lowest ionization stages, that cannot survive even at very low temperature. For instance, for $\alpha=2$ and the extreme corresponding value of $x_{\mathrm{b}}=\alpha+1 / 2$, the mean charge is already +4 for oxygen and +6 for iron at $T=10^{4} \mathrm{~K}$. At high temperature, the mean charge can typically be changed by a few units, the effect being more important in the temperature range where the mean charge changes rapidly with temperature in the Maxwellian case.
The same behavior is seen for all elements (Fig. 18). One notes that the effect of the Hybrid distribution generally decreases with $Z$. Again this is a consequence of the same behavior observed on the ionization rates (see Fig. 7).

A remarkable effect of the Hybrid distribution is that the mean charge is not always a monotonous function of temperature, in the low temperature regime. This is clearly apparent in Figs. 17 and 18 for $10^{4} \mathrm{~K} \leq T \leq 10^{5} \mathrm{~K}$ and $x_{\mathrm{b}}=10$. This phenomenon can only occur when the dielectronic rate dominates the total recombination rate and in the temperature range where this rate increases with temperature. In that case, the density ratio of two adjacent ions, $N_{Z, z+1} / N_{Z, z}$, can decrease with temperature provided that the ionization rate of $Z^{+z}$ increases less rapidly with temperature than the recombination rate of the adjacent ion $Z^{+(z+1)}$ (Eq. (18)). This usually does not occur in the Maxwellian case, but can occur in the Hybrid case, due to the flatter temperature dependence of the ionization rates for this type of distribution. For instance, for $3 \times 10^{4} \mathrm{~K} \leq T \leq 7 \times 10^{4} \mathrm{~K}$, the ionization rate of $\mathrm{O}^{+2}$ is increased by a factor of 2.5 for an Hybrid distribution with $x_{\mathrm{b}}=10$ (Fig. 8), whereas the total recombination 

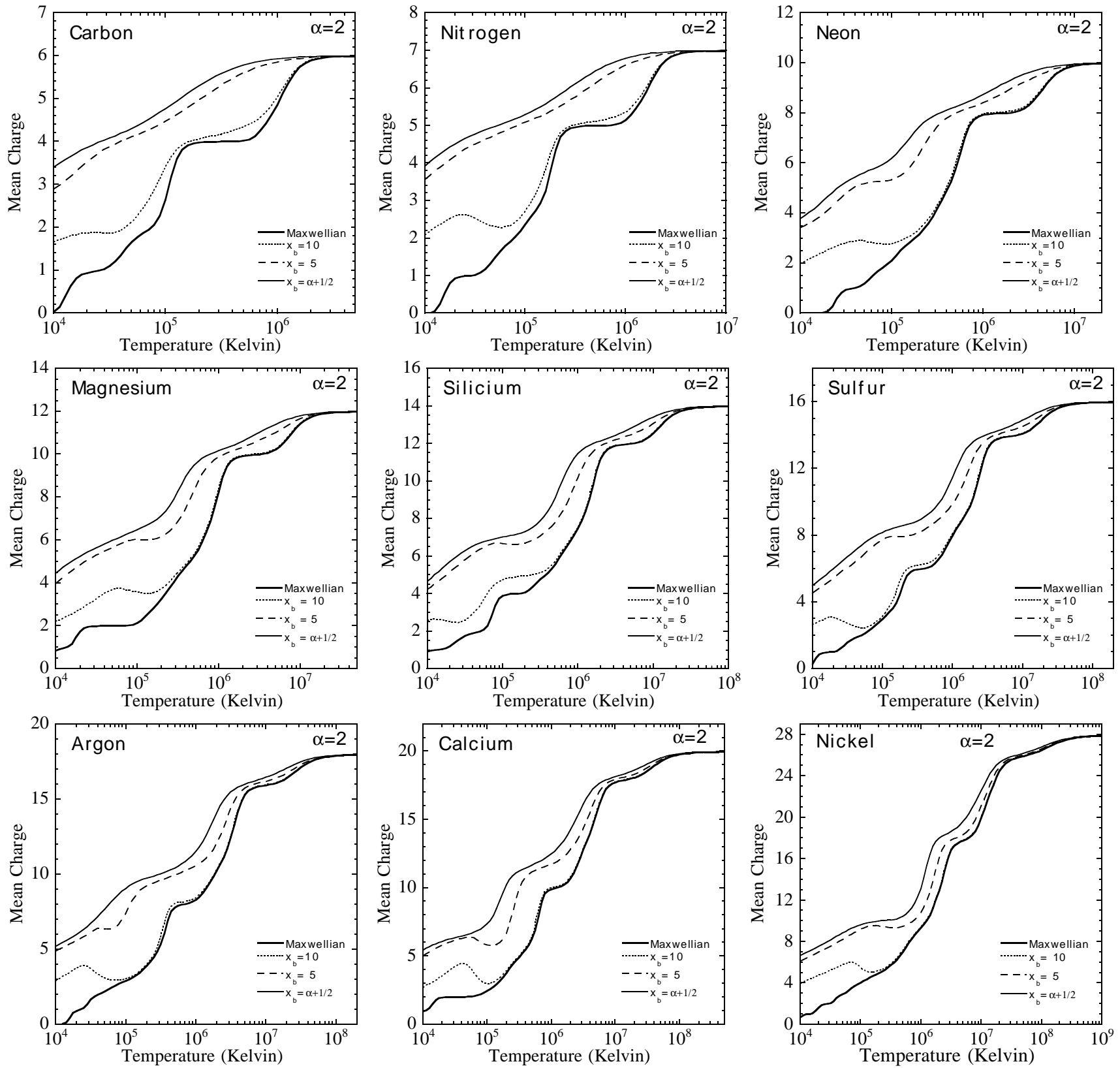

Fig. 18. Mean electric charge versus temperature for the Hybrid electron distribution (black thin lines) compared to the Maxwellian distribution (black thick lines). The slope parameter $\alpha$ of the Hybrid distribution is fixed to 2, and the $x_{\mathrm{b}}$ values are as in Fig. 17. Each panel corresponds to an element and is labelled accordingly.

rate of $\mathrm{O}^{+3}$ is increased by a slightly larger factor of 2.7 (see the corresponding grey line in Fig. 16, as seen above for $x_{\mathrm{b}}=10$ the total rate is basically unchanged compared to the Maxwellian case). The mean charge, which is around $\langle z\rangle=2.5$, is thus dominated by the behavior of these ions and decreases in that temperature range.

\subsection{Non-equilibrium ionization (NEI)}

Collisional Ionization Equilibrium (CIE) is not always achieved. For example, in adiabatic supernova remnants, the ionization timescale is longer than the dynamical timescale, so that the plasma is underionized compared to the equilibrium case. In non-equilibrium conditions, the ionization state of the gas depends on the thermodynamic history of the shocked gas (temperature, density) and time elapsed since it has been shocked.

The time evolution of the ionic fractions is given by:

$$
\begin{aligned}
\frac{\mathrm{d} X_{Z, z}}{\mathrm{~d} t}= & n_{\mathrm{e}}\left[C_{\mathrm{I}}^{Z, z-1} X_{Z, z-1}+\alpha_{R}^{Z, z+1} X_{Z, z+1}\right. \\
& \left.-\left(C_{I}^{Z, z}+\alpha_{R}^{Z, z}\right) X_{Z, z}\right] \\
\text { with } X_{Z, z}= & \frac{N_{Z, z}}{\sum_{i} N_{Z, i}} .
\end{aligned}
$$

To estimate the effects of a Hybrid electron distribution on the ionization in non-equilibrium ionization conditions, 

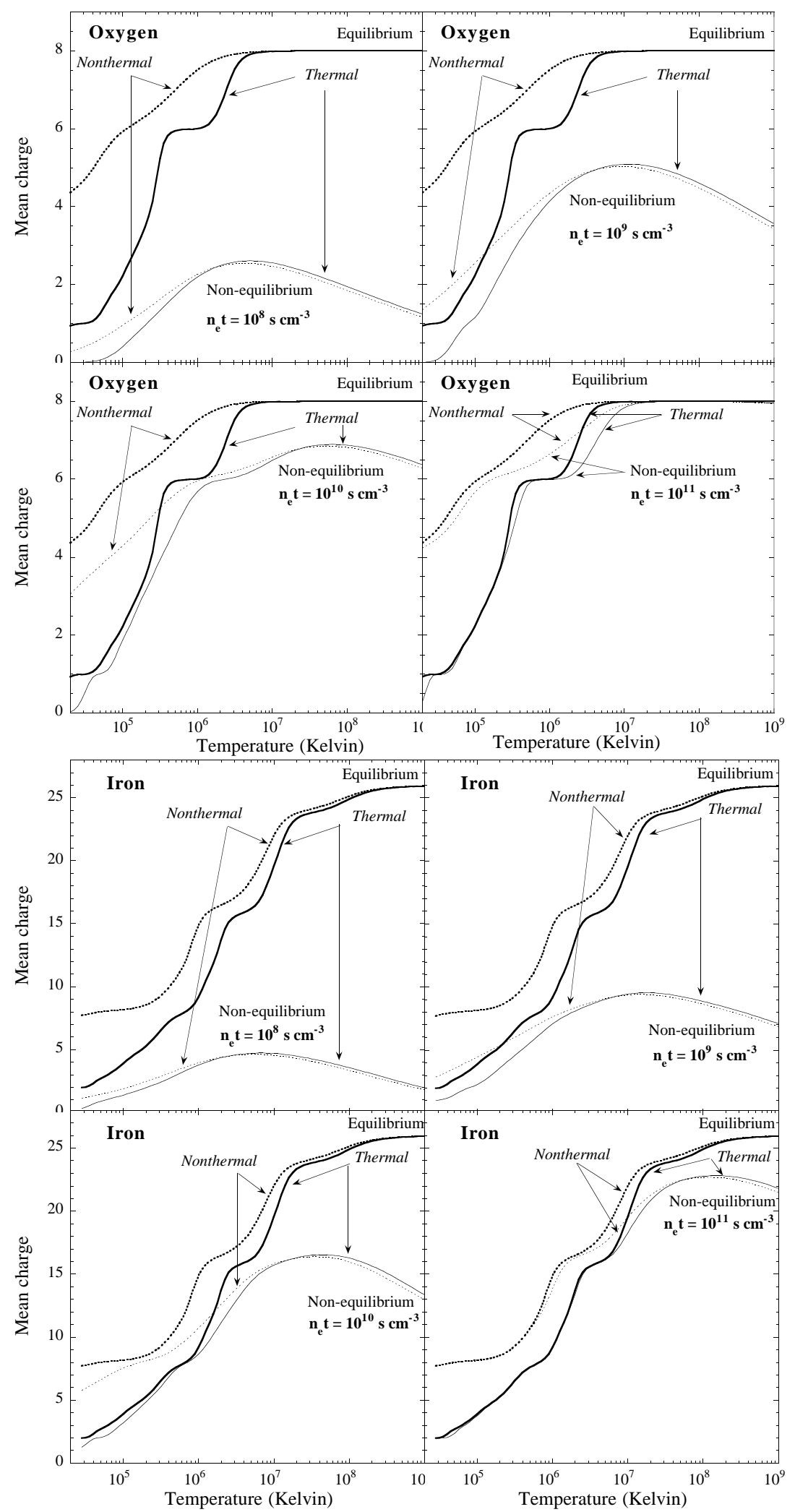

Fig. 19. Mean electric charge versus temperature for oxygen (top panels) and iron (bottom panels) for different ionization timescales (thin lines) compared to equilibrium (bold lines) and for two extreme electron distributions, namely Maxwellian (solid lines) and Hybrid (dotted lines) for $\alpha=2$ and $x_{\mathrm{b}}=\alpha+1 / 2$.

we assume that the gas has been suddently heated to a given temperature, which stays constant during the evolution. The ionization timescale depends then on $\int n_{\mathrm{e}} \mathrm{d} t$, where $n_{\mathrm{e}}$ is the number density of electrons and 
$t$ the time elapsed since the gas has been heated. Within this assumption, the coupled system of rate equations can be resolved using an exponentiation method (e.g., Hughes \& Helfand 1985).

For different ionization timescales (up to equilibrium), we computed the variation with temperature of the mean electric charge of oxygen and iron in two extreme cases of the electron distribution: Maxwellian and Hybrid with $x_{\mathrm{b}}=\alpha+1 / 2$ and $\alpha=2$. For small ionization timescales $\left(n_{\mathrm{e}} t \simeq 10^{8}-10^{9} \mathrm{~s} \mathrm{~cm}^{-3}\right)$, the effect of the Hybrid distribution on the mean electric charge is small, it increases with the ionization timescale and is maximum at equilibrium as is illustrated for oxygen and iron in Fig. 19. As in the equilibrium case, the effect from non-thermal electrons is always more important at low temperature and vanishes at high temperature. Note that the mean electric charge is slightly larger at high temperature for the thermal population than for the non-thermal one, as a consequence of the decrease of the ionization cross section at very high energy.

\section{Conclusions}

We have studied the effect on the ionization and recombination rates, as well as on the ionization balance, of a non-thermal electron distribution, as expected in the vicinity of strong shocks.

The electron distribution is modelled by a Maxwellian distribution at low energy up to a break energy, and by a power-law distribution at higher energy. It is caracterised by the three parameters $k T$ (the temperature of the Maxwellian part), $x_{\mathrm{b}}$ the reduced energy break, and $\alpha$ the slope of the power-law component. We only considered the parameter range where $x_{\mathrm{b}}>\alpha+1 / 2$ which corresponds to an enhanced high energy tail. All the behaviors outlined are only valid for this range of parameters.

We provide exact formulae of the ionization rates for this Hybrid electron distribution in the Appendix, and approximate estimates of the radiative recombination rates (Eqs. (12) and (13)) and of the dielectronic recombination rates (Eq. (16)). The Hybrid rates depend on the ion considered and on the parameters $k T, \alpha$ and $x_{\mathrm{b}}$. Computer codes are available on request.

For the parameter range considered, the proportion of electrons at high energies and the mean energy of the distribution is a monotonic function of $x_{\mathrm{b}}$ and $\alpha$. As expected, the modification of the rates for the Hybrid distribution, as compared to the Maxwellian distribution of the same temperature, increases with decreasing $x_{\mathrm{b}}$ (with a threshold at about $x_{\mathrm{b}} \sim 10-20$, higher for lower temperature) and decreasing $\alpha$.

The impact of the Hybrid electron distribution on the ionization rates depends on how the high energy tail affects the proportion of electrons above the ionization potential $E_{\mathrm{I}}$. The Hybrid rates are increased with respect to the Maxwellian rates except at very high temperature. The enhancement factor depends on the temperature, mostly via the factor $E_{\mathrm{I}} / k T$, and increases dramatically with decreasing temperature. For a given ion, it is always important at $T^{*}$, the temperature of maximum ionization fraction for a Maxwellian distribution under ionization equilibrium, where it can reach several orders of magnitude.

The effect of the hybrid distribution on the dielectronic rate depends on the position of the resonance energies $E_{\mathrm{DR}}$ as compared to the power-law energy break. The dielectronic rate can only be increased if $k T<E_{\mathrm{DR}} /(\alpha+1 / 2)$. At $T^{*}$ the enhancement factor is typically less than an order of magnitude. At high temperature, the dielectronic recombination rate is slightly decreased (by typically $10 \%$ at most). The effect of the hybrid distribution on the radiative recombination rates is only of the order of a few $10 \%$ at most.

The ionization balance is affected significantly, whereas the effect is smaller in ionizing NIE plasmas. The plasma is always more ionized for a Hybrid electron distribution than for a Maxwellian distribution. The effect is more important at low temperature, and a clear signature of the Hybrid distribution is the disappearance of the lowest ionization stages, which cannot survive even at very low temperature.

Acknowledgements. We would like to thank Jean Ballet for a careful reading of the manuscript.

\section{Appendix A: Ionization rates}

\section{A.1. Direct ionization (DI)}

For the direct ionization (DI) cross sections we chose the fitting formula proposed by Arnaud \& Rothenflug (1985) from the work of Younger (1981):

$$
\begin{aligned}
\sigma_{\mathrm{DI}}(E)= & \sum_{j} \frac{1}{u_{j} I_{j}^{2}}\left[A_{j} U_{j}+B_{j} U_{j}^{2}+C_{j} \ln \left(u_{j}\right)+D_{j} \frac{\ln \left(u_{j}\right)}{u_{j}}\right] \\
& \text { with } u_{j}=\frac{E}{I_{j}} ; \quad U_{j}=1-\frac{1}{u_{j}} .
\end{aligned}
$$

The sum is performed over the subshells $j$ of the ionizing ion. $E$ is the incident electron energy and $I_{j}$ is the collisional ionization potential for the level $j$ considered.

The parameters $A_{j}, B_{j}, C_{j}, D_{j}$ (in units of $10^{-14} \mathrm{~cm}^{2} \mathrm{eV}^{2}$ ) and $I_{j}$ (in $\mathrm{eV}$ ) are taken from the works of Arnaud \& Raymond (1992) for iron, and of Arnaud \& Rothenflug (1985) for the others elements. The parameters for elements not considered in these works are given in Mazzotta et al. (1998).

\section{A.1.1. The Maxwellian electron distribution}

For a Maxwellian electron distribution, Arnaud \& Rothenflug (1985) obtained according to Eqs. (1), (8) and (A.1), the rate:

$C_{\mathrm{DI}}^{\mathrm{M}}(T)=\frac{6.692 \times 10^{7}}{(k T)^{3 / 2}} \sum_{j} \frac{\mathrm{e}^{-x_{j}}}{x_{j}} F_{\mathrm{DI}}^{\mathrm{M}}\left(x_{j}\right) \quad \mathrm{cm}^{3} \mathrm{~s}^{-1}$ 
where

$$
\begin{aligned}
x_{j}= & \frac{I_{j}}{k T} \\
F_{\mathrm{DI}}^{\mathrm{M}}\left(x_{j}\right)= & A_{j}\left[1-x_{j} f_{1}\left(x_{j}\right)\right] \\
& +B_{j}\left[1+x_{j}-x_{j}\left(2+x_{j}\right) f_{1}\left(x_{j}\right)\right] \\
& +C_{j} f_{1}\left(x_{j}\right)+D_{j} x_{j} f_{2}\left(x_{j}\right)
\end{aligned}
$$

where $k T$ and $I_{j}$ are in $\mathrm{eV}$. The summation is performed over the subshells $j$ of the ionizing ion. The mathematical functions, $f_{1}(x)=\mathrm{e}^{x} \int_{1}^{\infty} \frac{\mathrm{e}^{-t x}}{t} \mathrm{~d} t$, and $f_{2}(x)=$ $\mathrm{e}^{x} \int_{1}^{\infty} \frac{\mathrm{e}^{-t x}}{t} \ln (t) \mathrm{d} t$ can be computed from the analytical approximations given by Arnaud \& Rothenflug (1985) in their Appendix B.

\section{A.1.2. The Hybrid electron distribution}

Similar to the Hybrid electron distribution, the direct ionization rate $C_{\mathrm{DI}}^{\mathrm{H}}\left(T, x_{\mathrm{b}}, \alpha\right)$ is given by:

$$
\begin{aligned}
C_{\mathrm{DI}}^{\mathrm{H}}\left(T, x_{\mathrm{b}}, \alpha\right)= & C\left(x_{\mathrm{b}}, \alpha\right) \frac{6.692 \times 10^{7}}{(k T)^{3 / 2}} \\
& \times \sum_{j} G_{\mathrm{DI}}^{\mathrm{H}}\left(x_{j}, x_{\mathrm{b}}, \alpha\right) \quad \mathrm{cm}^{3} \mathrm{~s}^{-1} .
\end{aligned}
$$

The function $G_{\mathrm{DI}}^{\mathrm{H}}\left(x_{j}, x_{\mathrm{b}}, \alpha\right)$ depends on the position of the power-law break energy as compared to the ionization potential:

- For $x_{\mathrm{b}}>x_{j}$ :

$G_{\mathrm{DI}}^{\mathrm{H}}\left(x_{j}, x_{\mathrm{b}}, \alpha\right)$ is the sum of the contribution of the truncated Maxwellian component and the power-law component:

$$
\begin{aligned}
G_{\mathrm{DI}}^{\mathrm{H}}\left(x_{j}, x_{\mathrm{b}}, \alpha\right)= & \frac{\mathrm{e}^{-x_{j}}}{x_{j}} F_{\mathrm{DI}}^{\mathrm{M}}\left(x_{j}\right)-\frac{\mathrm{e}^{-x_{\mathrm{b}}}}{x_{j}} F_{\mathrm{DI}}^{\prime}\left(x_{j}, x_{\mathrm{b}}\right) \\
& +\mathrm{e}^{-x_{\mathrm{b}}} F_{\mathrm{DI}}^{\mathrm{PL}}\left(u_{\mathrm{b}_{j}}, \alpha\right)
\end{aligned}
$$

where:

$$
\begin{aligned}
u_{\mathrm{b}_{j}}= & \frac{x_{\mathrm{b}}}{x_{j}} \\
F_{\mathrm{DI}}^{\prime}\left(x_{j}, x_{\mathrm{b}}\right)= & A_{j}\left[1-x_{j} f_{1}\left(x_{\mathrm{b}}\right)\right] \\
& +B_{j}\left[1+\frac{x_{j}}{u_{\mathrm{b}_{j}}}-x_{j}\left(2+x_{j}\right) f_{1}\left(x_{\mathrm{b}}\right)\right] \\
& +C_{j}\left[f_{1}\left(x_{\mathrm{b}}\right)+\ln \left(u_{\mathrm{b}_{j}}\right)\right] \\
& +D_{j}\left[x_{j} f_{2}\left(x_{\mathrm{b}}\right)+\ln \left(u_{\mathrm{b}_{j}}\right) x_{j} f_{1}\left(x_{\mathrm{b}}\right)\right] \\
F_{\mathrm{DI}}^{\mathrm{PL}}\left(u_{\mathrm{b}_{j}}, \alpha\right)= & A_{j}\left[\frac{u_{\mathrm{b}_{j}}}{\alpha-1 / 2}-\frac{1}{\alpha+1 / 2}\right] \\
& +B_{j}\left[\frac{u_{\mathrm{b}_{j}}}{\alpha-1 / 2}-\frac{2}{\alpha+1 / 2}+\frac{u_{\mathrm{b}_{j}}^{-1}}{\alpha+3 / 2}\right] \\
& +\frac{C_{j} u_{\mathrm{b}_{j}}}{\alpha-1 / 2}\left[\ln \left(u_{\mathrm{b}_{j}}\right)+\frac{1}{\alpha-1 / 2}\right] \\
& +\frac{D_{j}}{\alpha+1 / 2}\left[\ln \left(u_{\mathrm{b}_{j}}\right)+\frac{1}{\alpha+1 / 2}\right] .
\end{aligned}
$$

- For $x_{\mathrm{b}}<x_{j}$ :

Only the power-law component contributes of the electron distribution to the rate:

$G_{\mathrm{DI}}^{\mathrm{H}}\left(x_{j}, x_{\mathrm{b}}, \alpha\right)=\mathrm{e}^{-x_{\mathrm{b}}}\left(\frac{x_{\mathrm{b}}}{x_{j}}\right)^{\alpha+\frac{1}{2}} f_{\mathrm{DI}}(\alpha)$

where

$$
\begin{aligned}
f_{\mathrm{DI}}(\alpha)= & \frac{A_{j}}{\alpha^{2}-1 / 4}+\frac{2 B_{j}}{\left(\alpha^{2}-1 / 4\right)(\alpha+3 / 2)} \\
& +\frac{C_{j}}{(\alpha-1 / 2)^{2}}+\frac{D_{j}}{(\alpha+1 / 2)^{2}} .
\end{aligned}
$$

\section{A.2. Excitation autoionization (EA)}

For the excitation autoionization (EA) cross sections, we used the generalized formula proposed by Arnaud \& Raymond (1992):

$$
\begin{aligned}
\sigma_{\mathrm{EA}}(E)= & \frac{1}{u I_{\mathrm{EA}}}\left[A+B U+C U_{2}+D U_{3}+E \ln (u)\right] \\
& \text { with } u=\frac{E}{I_{\mathrm{EA}}} ; \quad U_{n}=1-\frac{1}{u^{n}}
\end{aligned}
$$

where $I_{\mathrm{EA}}$ is the excitation autoionization threshold and $\mathrm{E}$ is the incident electron energy.

The parameters $A, B, C, D, E$ (in units of $10^{-16} \mathrm{~cm}^{2} \mathrm{eV}$ ) and $I_{\mathrm{EA}}$ (in $\mathrm{eV}$ ) are taken from the works of Arnaud \& Rothenflug (1985) and Arnaud \& Raymond (1992). The parameters for elements not considered in these works are given in Mazzotta et al. (1998).

\section{A.2.1. The Maxwellian electron distribution}

The excitation autoionization rate for a Maxwellian distribution is:

$$
C_{\mathrm{EA}}^{\mathrm{M}}(T)=\frac{6.692 \times 10^{7} \mathrm{e}^{-x_{\mathrm{EA}}}}{(k T)^{1 / 2}} F_{\mathrm{EA}}^{\mathrm{M}}\left(x_{\mathrm{EA}}\right) \mathrm{cm}^{3} \mathrm{~s}^{-1}
$$

where

$$
\begin{aligned}
x_{\mathrm{EA}}= & \frac{I_{\mathrm{EA}}}{k T} \\
F_{\mathrm{EA}}^{\mathrm{M}}\left(x_{\mathrm{EA}}\right)= & A+B\left[1-x_{\mathrm{EA}} f_{1}\left(x_{\mathrm{EA}}\right)\right] \\
& +C\left[1-x_{\mathrm{EA}}+x_{\mathrm{EA}}^{2} f_{1}\left(x_{\mathrm{EA}}\right)\right] \\
& +D\left[1-\frac{x_{\mathrm{EA}}}{2}+\frac{x_{\mathrm{EA}}^{2}}{2}-\frac{x_{\mathrm{EA}}^{3}}{2} f_{1}\left(x_{\mathrm{EA}}\right)\right] \\
& +E f_{1}\left(x_{\mathrm{EA}}\right) .
\end{aligned}
$$

\section{A.2.2. The Hybrid electron distribution}

For the Hybrid electron distribution, the excitation autoionization rate $C_{\mathrm{EA}}^{\mathrm{H}}\left(T, x_{\mathrm{b}}, \alpha\right)$ is given by:

$$
\begin{aligned}
C_{\mathrm{EA}}^{\mathrm{H}}\left(T, x_{\mathrm{b}}, \alpha\right)= & C\left(x_{\mathrm{b}}, \alpha\right) \frac{6.692 \times 10^{7}}{(k T)^{1 / 2}} \\
& \times G_{\mathrm{EA}}^{\mathrm{H}}\left(x_{\mathrm{EA}}, x_{\mathrm{b}}, \alpha\right) \quad \mathrm{cm}^{3} \mathrm{~s}^{-1} .
\end{aligned}
$$


The function $G_{\mathrm{EA}}^{\mathrm{H}}\left(x_{\mathrm{EA}}, x_{\mathrm{b}}, \alpha\right)$ depends on the position of the power-law break energy as compared to the ionization potential:

- For $x_{\mathrm{b}}>x_{\mathrm{EA}}$ : $G_{\mathrm{EA}}^{\mathrm{H}}\left(x_{\mathrm{EA}}, x_{\mathrm{b}}, \alpha\right)$ is the sum of the contribution of the truncated Maxwellian component and the power-law component:

$$
\begin{aligned}
G_{\mathrm{EA}}^{\mathrm{H}}\left(x_{\mathrm{EA}}, x_{\mathrm{b}}, \alpha\right)= & \mathrm{e}^{-x_{\mathrm{EA}}} F_{\mathrm{EA}}^{\mathrm{M}}\left(x_{\mathrm{EA}}\right) \\
& -\mathrm{e}^{-x_{\mathrm{b}}} F_{\mathrm{EA}}^{\prime}\left(x_{\mathrm{EA}}, x_{\mathrm{b}}\right) \\
& +x_{\mathrm{b}} \mathrm{e}^{-x_{\mathrm{b}}} F_{\mathrm{EA}}^{\mathrm{PL}}\left(u_{\mathrm{EA}}, \alpha\right)
\end{aligned}
$$

where:

$$
\begin{aligned}
u_{\mathrm{EA}}= & \frac{x_{\mathrm{b}}}{x_{\mathrm{EA}}} \\
F_{\mathrm{EA}}^{\prime}\left(x_{\mathrm{EA}}, x_{\mathrm{b}}\right)= & A+B\left[1-x_{\mathrm{EA}} f_{1}\left(x_{\mathrm{b}}\right)\right] \\
& +C\left[1-\frac{x_{\mathrm{EA}}}{u_{\mathrm{EA}}}+x_{\mathrm{EA}}^{2} f_{1}\left(x_{\mathrm{b}}\right)\right] \\
& +D\left[1-\frac{x_{\mathrm{EA}}}{2 u_{\mathrm{EA}}^{2}}+\frac{x_{\mathrm{EA}}^{2}}{2 u_{\mathrm{EA}}}-\frac{x_{\mathrm{EA}}^{3}}{2} f_{1}\left(x_{\mathrm{b}}\right)\right] \\
& +E\left[\ln \left(u_{\mathrm{EA}}\right)+f_{1}\left(x_{\mathrm{b}}\right)\right] \\
F_{\mathrm{EA}}^{\mathrm{PL}}\left(u_{\mathrm{EA}}, \alpha\right)= & A\left[\frac{1}{\alpha-1 / 2}\right] \\
& +B\left[\frac{1}{\alpha-1 / 2}-\frac{u_{\mathrm{EA}}^{-1}}{\alpha+1 / 2}\right] \\
& +C\left[\frac{1}{\alpha-1 / 2}-\frac{u_{\mathrm{EA}}^{-2}}{\alpha+3 / 2}\right] \\
& +D\left[\frac{1}{\alpha-1 / 2}-\frac{u_{\mathrm{EA}}^{-3}}{\alpha+5 / 2}\right] \\
& +E\left[\frac{1}{(\alpha-1 / 2)^{2}}+\frac{\ln \left(u_{\mathrm{EA}}\right)}{\alpha-1 / 2}\right] .
\end{aligned}
$$

- For $x_{\mathrm{b}}<x_{\mathrm{EA}}$ :

Only the power-law component contributes of the electron distribution to the rate:

$$
G_{\mathrm{EA}}^{\mathrm{H}}\left(x_{\mathrm{EA}}, x_{\mathrm{b}}, \alpha\right)=x_{\mathrm{b}} \mathrm{e}^{-x_{\mathrm{b}}}\left(\frac{x_{\mathrm{b}}}{x_{\mathrm{EA}}}\right)^{\alpha-\frac{1}{2}} f_{\mathrm{EA}}(\alpha)
$$

where

$$
\begin{aligned}
f_{\mathrm{EA}}(\alpha)= & \frac{A}{\alpha-1 / 2}+\frac{B}{\alpha^{2}-1 / 4} \\
& +\frac{2 C}{(\alpha-1 / 2)(\alpha+3 / 2)} \\
& +\frac{3 D}{(\alpha-1 / 2)(\alpha+5 / 2)} \\
& +\frac{E}{(\alpha-1 / 2)^{2}} .
\end{aligned}
$$

\section{A.3. Total ionization rates $(D I+E A)$}

The total ionization rate $C_{\mathrm{I}}^{\mathrm{H}}\left(T, x_{\mathrm{b}}, \alpha\right)$ is obtained by:

$C_{\mathrm{I}}^{\mathrm{H}}\left(T, x_{\mathrm{b}}, \alpha\right)=C_{\mathrm{DI}}^{\mathrm{H}}\left(T, x_{\mathrm{b}}, \alpha\right)+C_{\mathrm{EA}}^{\mathrm{H}}\left(T, x_{\mathrm{b}}, \alpha\right)$.

\section{References}

Aldrovandi, S. M. V., \& Péquignot, D. 1973, A\&A, 25, 137

Allen, G. E., Keohane, J. W., Gotthelf, E. V., et al. 1997, ApJ, 487, L97

Arnaud, M., \& Rothenflug, R. 1985, A\&AS, 60, 425

Arnaud, M., \& Raymond, J. 1992, ApJ, 398, 394

Ballet, J., Luciani, J. F., \& Mora, P. 1989, A\&A, 218, 292

Baring, M. G., Ellison, D. C., Reynolds, S. P., Grenier, I. A., \& Goret, P. 1999, ApJ, 513, 311

Berezhko, E. G., \& Ellison, D. C. 1999, ApJ, 526, 385

Berezhko, E. G., \& Völk, H. J. 2000, APh, 14, 201

Blandford, R. D., \& Eichler, D. 1987, Phys. Rep., 154, 1

Bykov, A. M., \& Uvarov, Yu. A. 1999, JETP, 88, 465

Bykov, A. M., Bloemen, H., \& Uvarov, Yu. A. 2000a, A\&A, 362,886

Bykov, A. M., Chevalier, R. A., Ellison, D. C., \& Uvarov, Yu. A. 2000b, ApJ, 538, 203

Decourchelle, A., Ellison, D. C., \& Ballet, J. 2000, ApJ, 543, L57

Drury, L. O' C. 1983, Rep. Prog. Phys., 46, 973

Dzifcáková, E. 1992, Solar Phys., 140, 247

Dzifcáková, E. 1998, Solar Phys., 178, 317

Dzifcáková, E. 2000, Solar Phys., 196, 113

Ellison, D. C., Berezhko, E. G., \& Baring, M. G. 2000, ApJ, 540,292

Gaisser, T. K., Protheroe, R. J., \& Stanev, T. 1998, ApJ, 492, 219

Hughes, J. P., \& Helfand, D. J. 1985, ApJ, 291, 544

Hughes, J. P., Rakowski, C. E., \& Decourchelle, A. 2000, ApJ, 543, L61

Jones, F. C., \& Ellison, D. C. 1991, Space Sci. Rev., 58, 259

Kang, H., \& Jones, T. W. 1991, ApJ, 249, 439

Koyama, K., Petre, R., Gotthelf, E. V., et al. 1995, Nature, 378,255

Koyama, K., Kinugasa, K., Matsuzaki, K., et al. 1997, PASJ, 49, L7

Laming, J. M. 2001, ApJ, 546, 1149

Mazzotta, P., Mazzitelli, G., Colafrancesco, S., \& Vittorio, N. 1998, A\&AS, 133, 403

Owocki, S. P., \& Scudder, J. D. 1983, ApJ, 270, 758

Reynolds, S. P. 1996, ApJ, 459, L13

Reynolds, S. P. 1998, ApJ, 493, 375

Roussel-Dupré, R. 1980, Solar Phys., 68, 243

Sarazin, C. L. 1999, ApJ, 520, 529

Sarazin, C. L., \& Kempner, J. C. 2000, ApJ, 533, 73

Seely, J. F., Feldman, U., \& Doschek, G. A. 1987, ApJ, 319, 541

Slane, P., Gaensler, B. M., Dame, T. M., et al. 1999, ApJ, 525, 357

Slane, P., Hughes, J. P., Edgar, R. J., et al. 2001, ApJ, 548, 814

Sturner, S. J., Skibo, J. G., Dermer, C. D., \& Mattox, J. R. 1997, ApJ, 490, 619

Tawara, H., Kato, T., \& Ohnishi, M. 1985, IPPJ-AM-37, Institute of Plasma Physics, Nagoya University

Younger, S. M. 1981, J. Quant. Spectrosc. Radiat. Transfer, 26,329 